\title{
Formalizing insect morphological data: a model-based, extensible insect anatomy ontology and its potential applications in biodiversity research and informatics
}

\author{
Jennifer C. Girón*, \\ Department of Entomology, Purdue University, West Lafayette, Indiana, USA \\ Natural Science Research Laboratory, Museum of Texas Tech University, Lubbock, Texas, USA. \\ entiminae@gmail.com; jgirondu@purdue.edu; jennifer.giron@ttu.edu \\ https://orcid.org/0000-0002-0851-6883
}

\section{Sergei Tarasov}

Finnish Museum of Natural History, Pohjoinen Rautatiekatu 13, FI-00014 Helsinki, Finland. sergei.tarasov@helsinki.fi https://orcid.org/0000-0001-5237-2330

\section{Luis Antonio González Montaña}

Universidad de los Llanos, Facultad de Ciencias Básicas e Ingeniería, Villavicencio, Meta, Colombia. luis.gonzalez.montana@unillanos.edu.co; lagonzalezmo@unal.edu.co https://orcid.org/0000-0002-9136-9932

\author{
Nicolas Matentzoglu \\ Semanticly Ltd, London, UK. \\ nicolas.matentzoglu@gmail.com \\ https://orcid.org/0000-0002-7356-1779
}

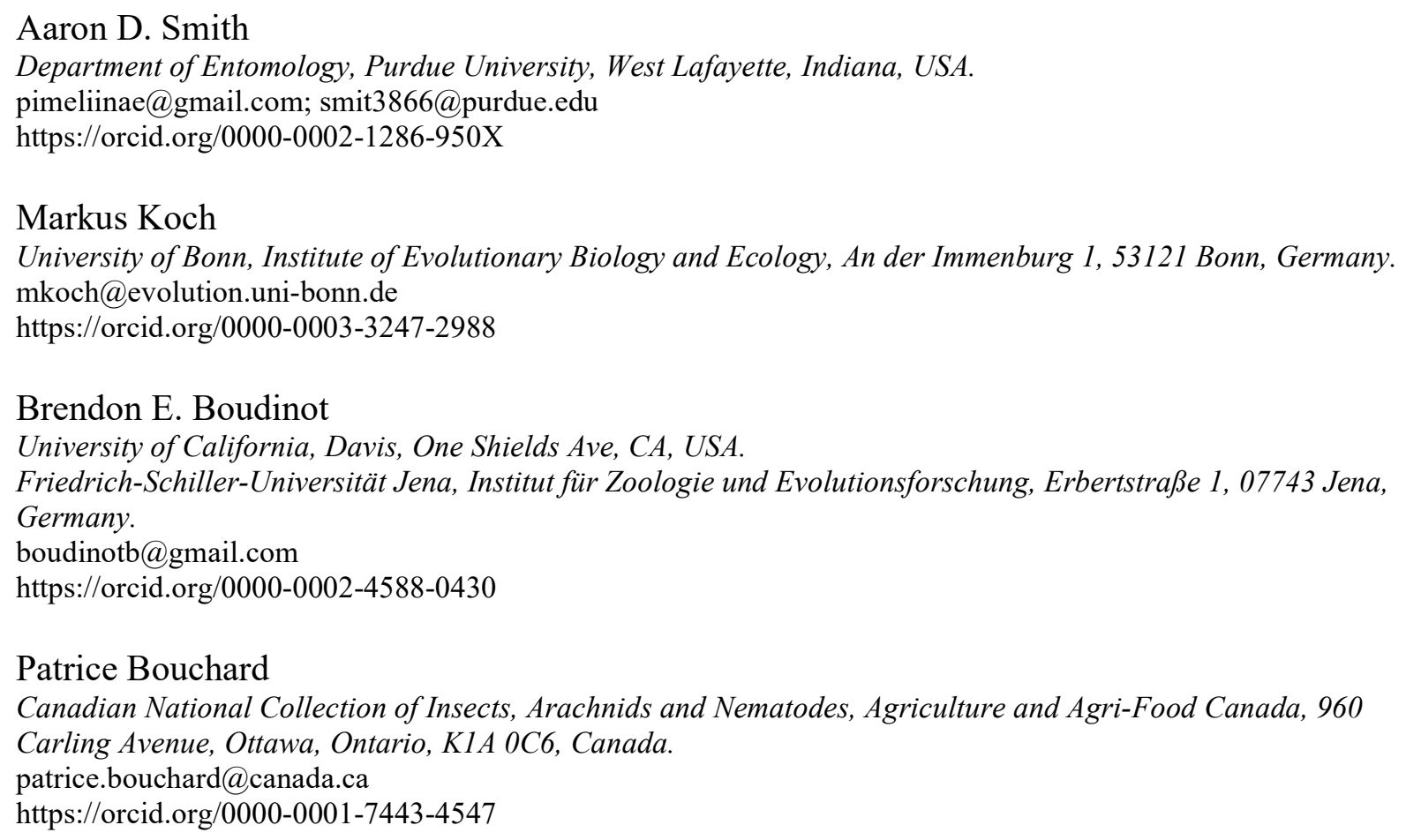


University of California, Riverside, Entomology Department, 900 University Ave. Riverside, CA, USA. burks.roger@gmail.com; roger.burks@ucr.edu https://orcid.org/0000-0003-3032-7939

\section{Lars Vogt}

TIB Leibniz Information Centre for Science and Technology, Welfengarten 1B, 30167 Hannover, Germany. lars.m.vogt@googlemail.com https://orcid.org/0000-0002-8280-0487

\section{Matthew Yoder}

University of Illinois, Illinois Natural History Survey, Champaign, IL, USA. diapriid@gmail.com https://orcid.org/0000-0002-5640-5491

\section{David Osumi-Sutherland}

European Bioinformatics institute (EMBL-EBI) WellcomeTrust Genome Campus, Cambridge UK. davidos@ebi.ac.uk https://orcid.org/0000-0002-7073-9172

\section{Frank Friedrich}

Universität Hamburg, institut fur Spezielle Zoologie, Hamburg, Germany. frank.friedrich@uni-hamburg.de

\section{Rolf Beutel}

Friedrich-Schiller-Universität Jena, Institut für Zoologie und Evolutionsforschung, Erbertstraße 1, 07743 Jena, Germany. rolf.beutel@uni-jena.de https://orcid.org/0000-0002-0433-7626

\section{István Mikó}

University of New Hampshire, Durham, NH, USA. istvan.miko@gmail.com; istvan.miko@unh.edu https://orcid.org/0000-0003-2938-9075

*Corresponding author: Jennifer C. Girón, entiminae@gmail.com Short title: Insect Anatomy Ontology 


\section{ABSTRACT}

The spectacular radiation of insects has produced a stunning diversity of phenotypes. During the last 250 years, research on insect systematics has generated hundreds of terms for naming and comparing those phenotypes. In its current form, this terminological diversity is presented in natural language and lacks formalization, which prohibits computer-assisted comparison using semantic web technologies. Here we propose a Model for Describing Insect Anatomical Structures (MoDIAS) which incorporates structural properties and positional relationships for standardized, consistent, and reproducible descriptions of insect phenotypes. We applied the MoDIAS framework in creating the ontology for the Anatomy of the Insect Skeleto-Muscular system (AISM). The AISM is the first general insect ontology that aims to cover all taxa by providing generalized, fully logical, and queryable, definitions for each term. It was built using the Ontology Development Kit (ODK), which maximizes interoperability with Uberon (Uberon multi-species anatomy ontology) and other basic ontologies, enhancing the integration of insect anatomy into the broader biological sciences. A template system for adding new terms, extending and linking the AISM to additional anatomical, phenotypic, genetic, and chemical ontologies is also introduced. The AISM is proposed as the backbone for taxon-specific insect ontologies and has potential applications spanning systematic biology and biodiversity informatics, allowing users to (1) use controlled vocabularies and create semi-automated computer-parsable insect morphological descriptions; (2) integrate insect morphology into broader fields of research, including ontology-informed phylogenetic methods, logical homology hypothesis testing, evo-devo studies, and genotype to phenotype mapping; and (3) automate the extraction of morphological data from the literature, enabling the generation of large-scale phenomic data, by facilitating the production and testing of informatic tools able to extract, link, 
annotate, and process morphological data. This system will allow for clear and semantically interoperable integration of insect phenotypes in biodiversity studies.

Keywords: Morphology, insects, biodiversity research, ontology development.

The ubiquitous distribution and stunning species richness of insects has generated a great diversity of phenotypes that fuel research in biodiversity, systematics, and various other biological fields. Roughly $90 \%$ of studies describing insect anatomy deal with structures related to the skeleto-muscular system (Deans et al. 2012a; Iyer et al. 2016; Adachi et al. 2020; Sommer 2020; Gotoh et al. 2021); the remaining 10\%, in general, deal with the nervous system (e.g., Loesel et al. 2013), the midgut (e.g., Monteiro et al. 2014), the endocrine system (e.g., Page Jr and Amdam 2007), fat bodies (e.g., de Oliveira and Cruz-Landim, 2003), etc. Thousands of morphological terms referring to the insect skeleto-muscular system have historically emerged due to several general processes: 1) most basic terms (e.g., head, wings, legs, etc.) have been borrowed from vertebrate anatomy due to functional or positional similarity; 2) some terms have been created de novo to name exclusive insect (or arthropod) structures (i.e., sclerite, tergite); 3) many terms have been repeatedly adopted across distant insect lineages to name similar structures located in similar areas of the body (e.g., cercus in Diplura vs. cercus in Hymenoptera; Snodgrass 1935); 4) the continuous reassessment of insect morphology in light of new comparative or phylogenetic data, constantly changes terms and their definitions; and 5) often, the definition of a term in subsequent studies, as in the "telephone game", suffers from interpretational deviations, thereby, producing a significantly different meaning that may eventually become widely adopted. 
The interplay of these term-generating processes brings two major persisting problems. First, numerous terms in the corpus seriously suffer from semantic ambiguities such as, homonymy (the same term is used for unrelated structures), polysemy (the same term is used for different but related -similar- structures) and synonymy (different terms with the same meaning) (Bolshoy and Lacková 2021). Second, many terms and definitions reflect the history of their usage rather than accurate anatomical concepts. Moreover, some terms refer to common spatiostructural properties, others refer to a common function or a common developmental or presumed common evolutionary origin, and some terms even refer to a mixture of these categories (Vogt et al. 2010). Consequently, interpreting and analyzing phenotypic data becomes unnecessarily difficult for non-experts and integrating phenotype data with other sources of data in the life sciences is very difficult and time-consuming.

These problems are compounded by our trend to see and characterize elements and developmental/evolutionary processes of the insect exoskeleton similarly to that of vertebrates (Snodgrass 1963), which resulted not only in misunderstanding of insect evolution and development, but also in an over-complicated system worsening the above-mentioned issues of insect morphological terminology. Bones, the main elements of the vertebrate skeleton, develop from well separated cell clusters into a complex scaffold mirroring the intricate 3D connections of cells with different function and origin (Bitsch and Bitsch 2002; Wang et al. 2017; Blumer 2021). Bones are connected to each other by different types of joints, whose accurate functioning requires the interplay of unrelated elements, including ligaments, articular cartilages, and synovial fluid (Blumer 2021). 
In comparison, the insect exoskeleton, the cuticle, is a simple, acellular product of the single-layered outer epithelium, the epidermis (Hall 1975; Adler 2017; Denk-Lobnig and Martin 2020) and its movable elements, the sclerites, are only stiffer regions of the cuticle that are surrounded by more flexible ones of the same origin (conjunctivae), granting mobility (Fig. 1). Therefore, unlike the vertebrate system, the insect skeleto-muscular system should be simply modeled using clearly identifiable and consistently organized building blocks of the continuous cuticle (Fig. 2). These building blocks — sclerites, conjunctiva, and formative elements (Klass 2008; Klass and Matushkina 2012); Table 1) — can also be used as anatomical landmark entities (i.e., disjointed intrinsically identifiable anatomical entities; Young 1993) for identifying units of comparison across different species (i.e., non-evolutionary comparative homology assessment; Vogt 2017). It is worth pointing out that the distinction between building blocks is not always clear, since semi-membranous areas also occur (e.g., often parts of the epipharynx, the wing articulation), so everything is more or less a continuum (Fig. 2), with (gradually) different degrees of sclerotization. The concept of "building blocks" (i.e., unambiguously defined sclerites), may be justified for pragmatic reasons in most cases, but it is still a simplification. 


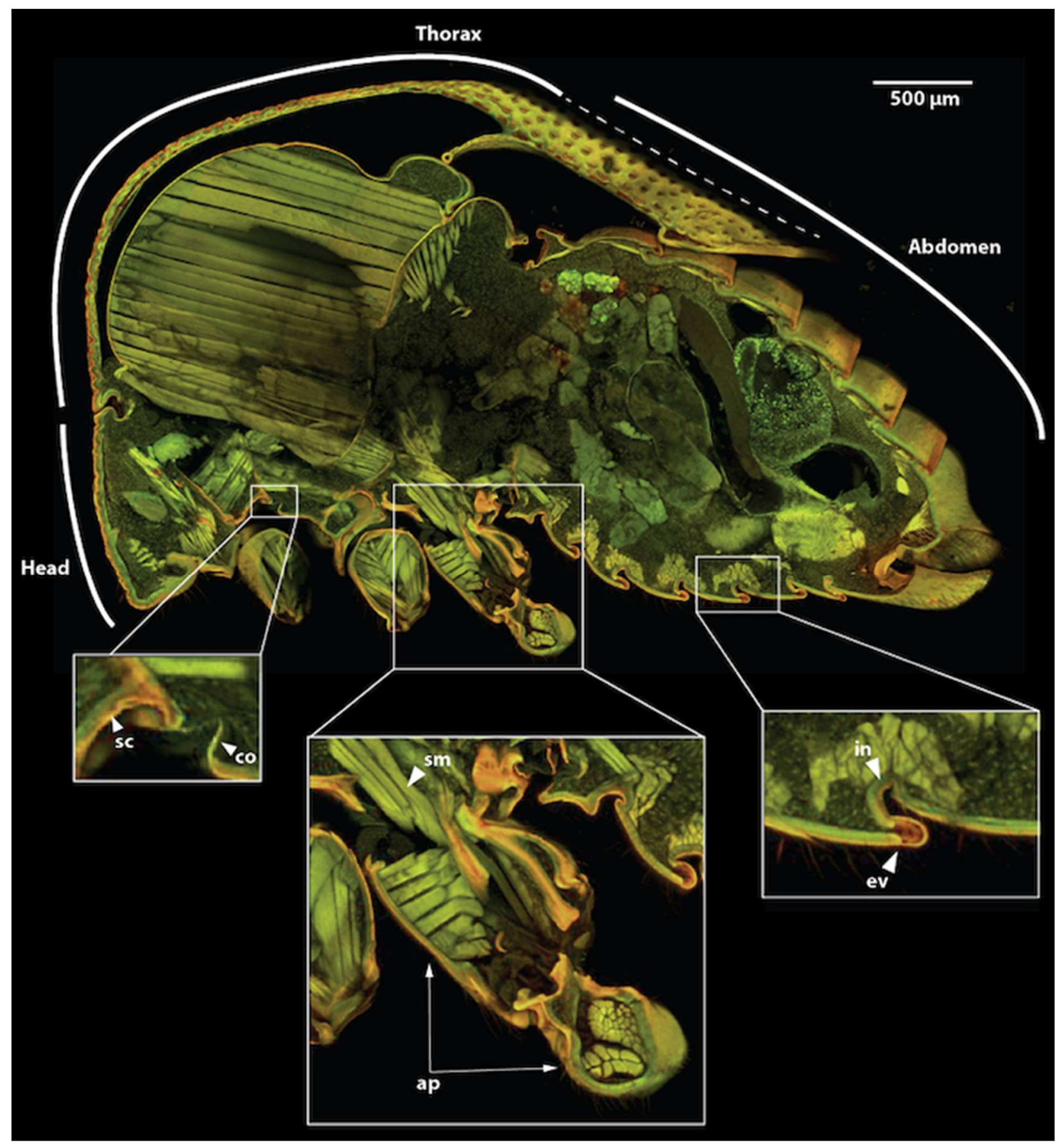

Figure 1. Autofluorescence-based CLSM micrograph showing the general structure of a sagittal section of the insect integument in an adult treehopper, genus Ceresa sp. (Membracidae). Excitation wavelength: 488, emission wavelengths: 500-580 pseudocolor green for conjunctivae, muscles, and other soft structures and 580-700 pseudocolor red for sclerotized components. 
Abbreviations: sc: sclerite; co: conjunctiva; sm: skeletal muscle; ap: appendage; in: invagination; ev: evagination.

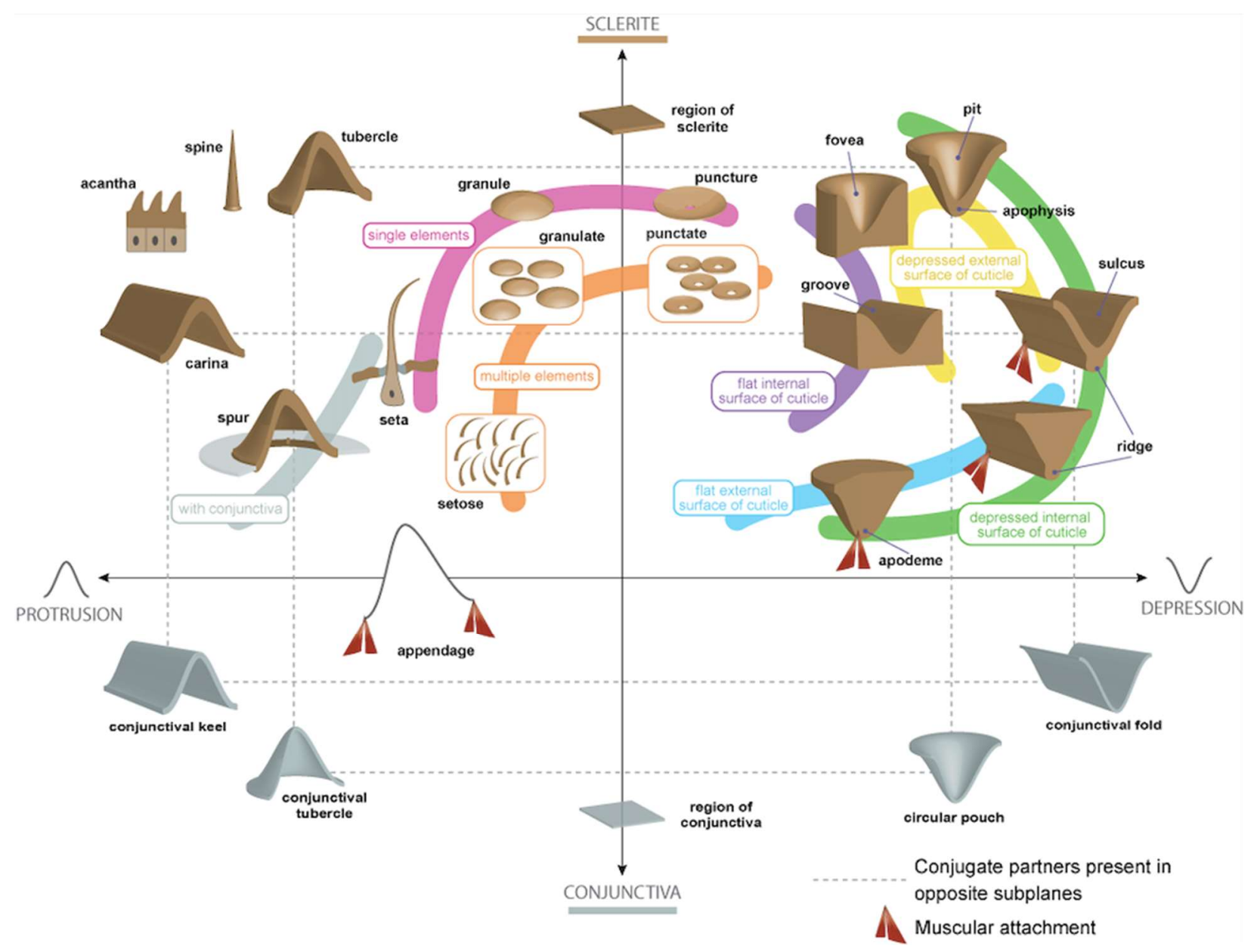

Figure 2. Schematic representation of some of the structural components of MoDIAS: a conceptual Model for Describing Insect Anatomical Structures. The two principal structural properties that characterize the insect cuticle are: 1) degree of flexibility (y axis), ranging from sclerite (stiff, at the top) to conjunctiva (flexible, at the bottom), and 2) degree of curvature (x axis; protrusion -left- to flat -center- to depression -right-); the degree of curvature of the external and internal surfaces of cuticular protrusions and depressions can be different: when both run in parallel, they form hollow protrusions (top left subplane, e.g., carina, tubercle) or hollow 
depressions (top right subplane, yellow band e.g., pit, sulcus); the external surface can be depressed with the internal surface flat (top right subplane, purple band, e.g., fovea, groove); the external surface can be flat with the internal surface depressed (top right subplane, blue band, e.g., ridge, apodeme). Additional properties: quantity (single vs. multiple elements; pink and orange bands, respectively); shape can be observed throughout each subplane (e.g., sclerotized protrusions can range from elongated - carina- to rounded -tubercle-); same for depressions (elongate -groove- vs. rounded -fovea-).

Table 1. Cuticular elements and structural properties used in the AISM.

\begin{tabular}{|c|c|c|}
\hline Subclass & Definition & URI \\
\hline region of cuticle & $\begin{array}{l}\text { The region of the insect integument } \\
\text { (UBERON:6007284) that is part of chitin-based } \\
\text { cuticle (UBERON:0001001) }\end{array}$ & AISM:0000174 \\
\hline sclerite & $\begin{array}{l}\text { The region of the cuticle (AISM:0000174) that is } \\
\text { less flexible than the neighboring conjunctiva(e) } \\
\text { (conjunctiva(e) (AISM:0000004) that the sclerite } \\
\text { is continuous with) }\end{array}$ & AISM:0000003 \\
\hline conjunctiva & $\begin{array}{l}\text { The region of the cuticle (AISM:0000174) that is } \\
\text { more flexible than the neighboring sclerite(s) } \\
\text { (AISM:0000003) (sclerite(s) that the conjunctiva } \\
\text { is continuous with) }\end{array}$ & AISM:0000004 \\
\hline $\begin{array}{l}\text { cuticular } \\
\text { depression }\end{array}$ & $\begin{array}{l}\text { The region of the cuticle that corresponds to a } \\
\text { concave surface }\end{array}$ & AISM:0000005 \\
\hline
\end{tabular}


cuticular

invagination

cuticular

protrusion

cuticular

evagination

anatomical

region
The region of cuticle (AISM:0000174) that

corresponds with an invagination of the single

layer epidermis (epithelial fold;

UBERON:0005157). The cuticular invagination

sometimes corresponds to a cuticular depression

(concavity on the surface of the cuticle;

AISM:0000005)

The region of the cuticle that corresponds to a

convex surface

The region of cuticle (AISM:0000174) that

corresponds with an evagination of the cuticle

and the single layer epidermis (epidermal fold;

UBERON:0005157). The cuticular evagination

usually corresponds to a cuticular protrusion

(convexity on the surface of the cuticle;

AISM:0000008)

A 3D region in space without well-defined

compartmental boundaries; for example, the

dorsal region of an ectoderm. [e.g., anterior

region (BSPO:0000071); lateral region

(BSPO:0000082); ventral margin

(BSPO:0000684)]
AISM:0000008

AISM:0000006

AISM:0000027

BSPO:0000070 
somatic muscle

A muscle structure (UBERON:0005090) of

UBERON:0014895

invertebrates whose origin and insertion sites are

in basal side of the epidermis or structures

derived from it. The simplest somatic muscles

consist of a single cell and associated

extracellular structures.

Ontologies have become a fundamental technology for semantic management and inference with biological knowledge (Smith et al. 2007; Balhoff et al. 2010; Deans et al. 2015; Dahdul et al. 2018; Tarasov 2019). An ontology is a logic-based representation of concepts and their relationships across a domain for modeling complex interactions in data (Deans et al., 2012a; Balhoff et al. 2013; Deans et al. 2015). In biology, ontologies serve two major purposes: they can be used as controlled vocabularies for stabilizing terminology and facilitate communication between scientists (Deans et al., 2012b), and as engines for inferring new complementary knowledge out of the encoded data. Therefore, ontology is a suitable technology for addressing the problems of understanding and interpreting terminology in insect morphology.

To date, there are seven ontologies dealing with the anatomy of different arthropod lineages, four of them dedicated to insects: Hymenoptera Anatomy Ontology (Yoder et al. 2010), Drosophila Anatomy Ontology (Osumi-Sutherland et al. 2013); Tribolium Ontology (Dönitz et al. 2013); and Mosquito Ontology (Topalis et al. 2008). However, given the narrow scope of each, none of them can be generally applied to insects as a whole. For the most part, these existing ontologies do not consider the interconnectedness of the whole cuticular system in their 
definitions, and those definitions tend to be idiosyncratic in the sense that they are taxon-specific and provide only textual/natural language definitions without much of a logical description, which prevents ontology-wide reasoning and inference.

One of the existing arthropod anatomy ontologies (Collembola Anatomy Ontology CLAO; González-Montaña 2021a; https://github.com/luis-gonzalez-m/Collembola) has been successfully used in the production of semantic-based morphological descriptions (GonzálezMontaña 2021b), demonstrating the potential for using anatomy ontologies in taxonomic research.

As a starting point towards a more stable, understandable, and interoperable terminology in insect morphology, in this study we provide a conceptual Model for Describing Insect Anatomical Structures (MoDIAS) at any developmental stage, in both formal and natural languages, and solely based on their structural properties and spatial relationships. We also provide the first universally applicable anatomy ontology for insects, the Anatomy Ontology of Skeleto-Muscular system (AISM), which is a formalized representation of MoDIAS that incorporates general terms for insect anatomy, including generalized definitions, while integrating them with other relevant ontologies. We provide ontology reasoning examples using the AISM and demonstrate its robustness and extensibility using the Ontology Development Kit (ODK). The AISM provides a computer-parsable controlled vocabulary for the insect skeletomuscular system with a broad range of applications, including serving as a backbone for taxonspecific ontologies, providing opportunities to data mine the existing literature, as well as 
producing semantically enhanced descriptions; it also has the potential for integration in evodevo research, phenotype to genotype mapping, and logical homology assessment analyses.

\section{METHODS}

\section{Model for Describing Insect Anatomical Structures (MoDIAS)}

The Model for Describing Insect Anatomical Structures (MoDIAS) incorporates structural properties and positional relationships to characterize anatomical structures used in morphological descriptions involving the insect skeleto-muscular system. We adopted anatomical concepts from Richards and Richards (1979), Snodgrass (1963), and Klass (2008) regarding the structural properties of the insect cuticle to define elementary building blocks (Fig. 2). For the positional relationships, we use terms referring to relative position of a given block along the body axes (dorsal, lateral, distal, etc.) and its connectedness to other structures (e.g., continuity, attachment). Using this model, each insect anatomical structure can be described and defined as one or more building blocks that are specifically related to other building blocks.

\section{Creating and editing the AISM}

The ontology for the Anatomy of the Insect Skeleto-Muscular system (AISM.owl) and accompanying file system were generated using the Ontology Development Kit (ODK, Matentzoglu et al. 2021; https://github.com/INCATools/ontology-development-kit) and edited with Protégé version 5.5.0 (Musen 2015). All the files are available on GitHub at https://github.com/insect-morphology/aism (Girón et al. 2021a). The ODK uses ROBOT-based workflows (Jackson et al. 2019; http://robot.obolibrary.org/) to automatically generate imports 
from related external ontologies including Uberon (Mungall et al. 2012) and the OBO relations ontology (Smith et al. 2005), and to drive quality control tests under continuous integration. It also provides a semi-automated release process supporting the generation of release products enhanced by the results of OWL reasoning.

Throughout this text we use bold lettering to indicate ontology classes, italics when referring to object properties, and use ID numbers to specify each. ID numbers are composed of the ontology prefix followed by colon and a number e.g., AISM:0000003. This ID represents a link (http://purl.obolibrary.org/obo/AISM_0000003) to an online version of the encoded information.

Following the principles proposed by MoDIAS, we created terms referring to the elementary building blocks of the insect skeleto-muscular system, as well as generalized terms from the glossary presented by Beutel et al. (2014). Each term has a label and a series of specific annotation properties including sensu (AISM:0000171), definition, contributor, and has exact synonym (Table. 2).

Table 2. Main annotation properties used in the AISM.

\begin{tabular}{lll}
\hline Annotation & AISM usage & URI \\
\hline label & A term indicated by a word or set of & $\underline{\text { https://www.w3.org/2000/01/rdf- }}$ \\
& words to unambiguously name an & $\underline{\text { schema\#label }}$ \\
& insect anatomical structure. &
\end{tabular}




\begin{tabular}{|c|c|c|}
\hline definition & A natural language statement to & http://purl.obolibrary.org/obo/IAO_0 \\
\hline & describe an insect anatomical & $\underline{000115}$ \\
\hline & structure, constructed by articulating & \\
\hline & the appropriate subclass of descriptors. & \\
\hline has exact & Alternative labels applied to the & http://www.geneontology.org/formats \\
\hline synonym & defined insect anatomical structure. & /oboInOwl\#hasExactSynonym \\
\hline & Should be accompanied by a sensu & \\
\hline & annotation. & \\
\hline sensu & Bibliographic reference with its & http://purl.obolibrary.org/obo/AISM_ \\
\hline & corresponding DOI (or other link to & $\underline{0000171}$ \\
\hline & it), and the textual definition of the & \\
\hline & term according to that reference. & \\
\hline contributor & The person who composed the & http://purl.obolibrary.org/dc/elements \\
\hline & definition or added the subclass of & $11.1 /$ contributor \\
\hline & descriptor & \\
\hline creation date & The date when the definition was & http://geneontology.org/formats/oboI \\
\hline & composed in year-month-day format. & $\underline{\text { nOwl\#creation date }}$ \\
\hline date_modified & Date on which the resource was & http://purl.org/dc/terms/modified \\
\hline & changed. & \\
\hline curator note & \& Additional comments to clarify or & http://purl.obolibrary.org/obo/IAO_0 \\
\hline & expand on the presented definition. & $\underline{000232}$ \\
\hline & Should be accompanied by & \\
\hline & contributor and creation_date. & \\
\hline
\end{tabular}




\begin{tabular}{ll}
\hline foaf:depiction & Associated image or images $\underline{\text { http://xmlns.com/foaf } / 0.1 / \text { depiction }}$ \\
& illustrating the structure being defined, \\
& linked by a DOI or URL.
\end{tabular}

Each AISM term is unambiguously labeled and formally represented by as many subclass of descriptors as necessary to clearly characterize the term using object properties and associated classes. Terms from existing general anatomy ontologies [e.g., Uberon multi-species anatomy ontology (Mungall et al. 2012); BFO: Basic Formal Ontology (Spear et al. 2016)], and supporting ontologies [e.g., BSPO: Biological Spatial Ontology (Dahdul et al. 2014); CARO: Common Anatomy Reference Ontology (Haendel et al. 2008); PATO: Phenotype And Trait Ontology (Gkoutos et al. 2005using; RO: Relation Ontology (Mungall et al. 2021)] were imported using the ODK.

An effort was made to maximize the inclusion of existing terms, avoiding duplication of existing object properties and general higher classes; we made sure that the class definitions offered in existing ontologies were compatible with the intended usage in the AISM before importing a class. Each term of the AISM is accompanied by a verbatim logical definition that translates each set of subclass of descriptors into natural language. Each definition in the AISM is intended to be broad enough to be applicable across Insecta, in a similar way as Uberon provides generalized definitions for animals (Mungall et al. 2012). As a convention, labels for muscles (which are given in English) and conjunctivae are given using their sites of attachment from proximal to distal, anterior to posterior, or dorsal to ventral. 
We used continuous with (RO:0002150) for sclerite-conjunctiva attachments, whereas adjacent to (RO:0002220) for sclerite-sclerite articulations. We also propose the object properties encircles (AISM:0000078) and encircled by (AISM:0000079) to annotate the relationship between ring sclerites and their corresponding conjunctivae (e.g., femur, antennomere).

\section{Reasoning}

We used a template (https://github.com/insectmorphology/aism/blob/master/AISM template_examples.tsv) to create AISM-based instances and definitions to demonstrate how the terms and generalized definitions provided in the AISM can be used to fit insect taxon-specific definitions more closely. In this template we represented different paired cuticular structures of the abdominal tergites as individuals (instances), for the orders Archaeognatha, Zygentoma, Dermaptera, Ephemeroptera (Baetidae), Hemiptera (Aphididae), Psocodea, and Coleoptera (Carabidae larva) (Table 3; see Fig. 3 for a schematic representation of these definitions). Using ROBOT (Jackson et al. 2019;

http://robot.obolibrary.org/) we generated an OWL file from this template, which included terms from the AISM and other ontologies. This template-based OWL file was then merged with the AISM. We ran a series of DL queries in Protégé, using ELK 0.5 as a reasoner on this merged ontology to verify the fit of the provided taxon-specific definitions with the terms and definitions available in the AISM (Table 4). The expectation was that the queries would return the appropriate instances, depending on the properties indicated in the template. 
1 Table 3. Example of template to specify new terms to include in AISM or AISM-derived ontologies. This template can be extended to

2 add more descriptors. See https://github.com/insect-morphology/aism/blob/master/AISM template examples.tsv.

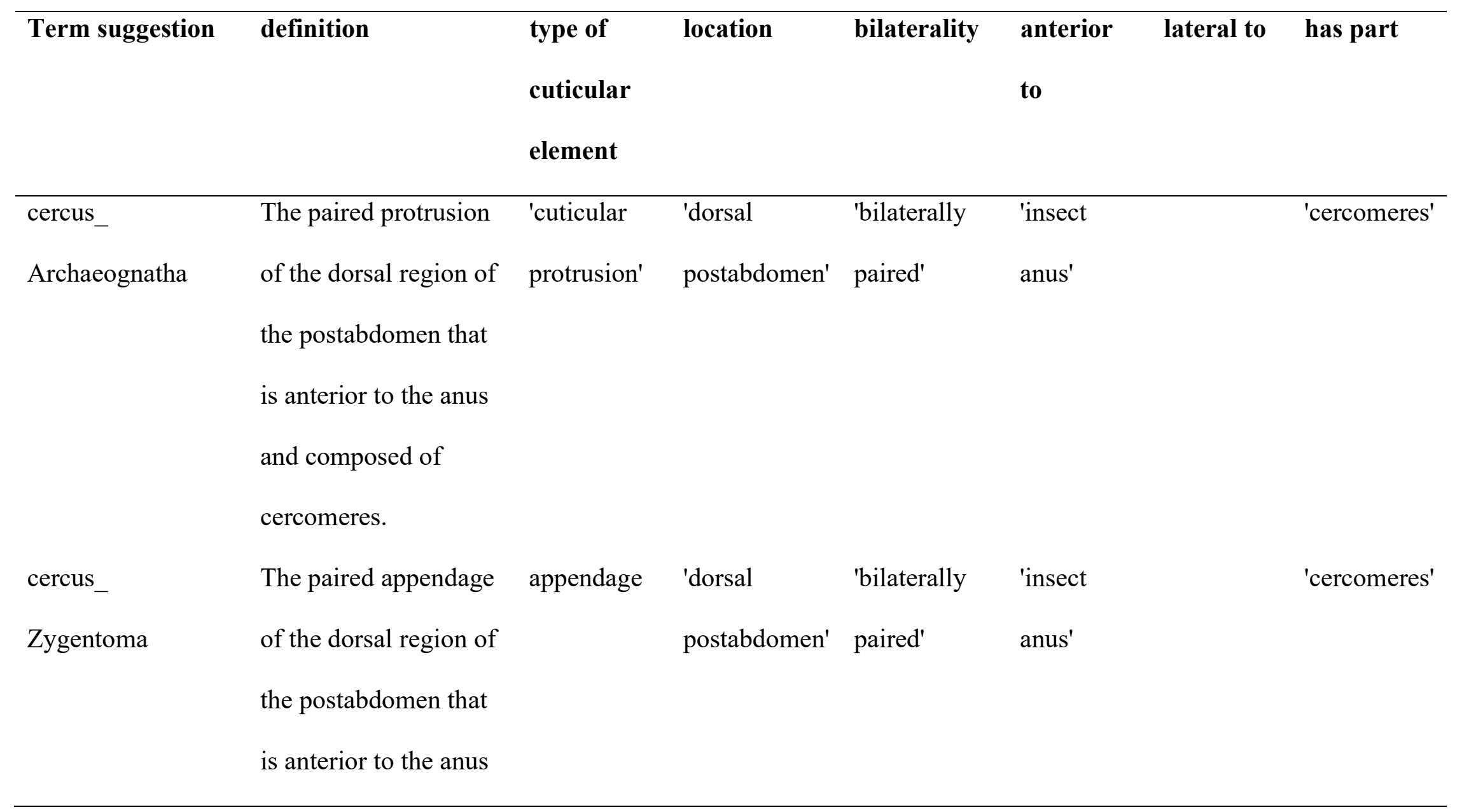




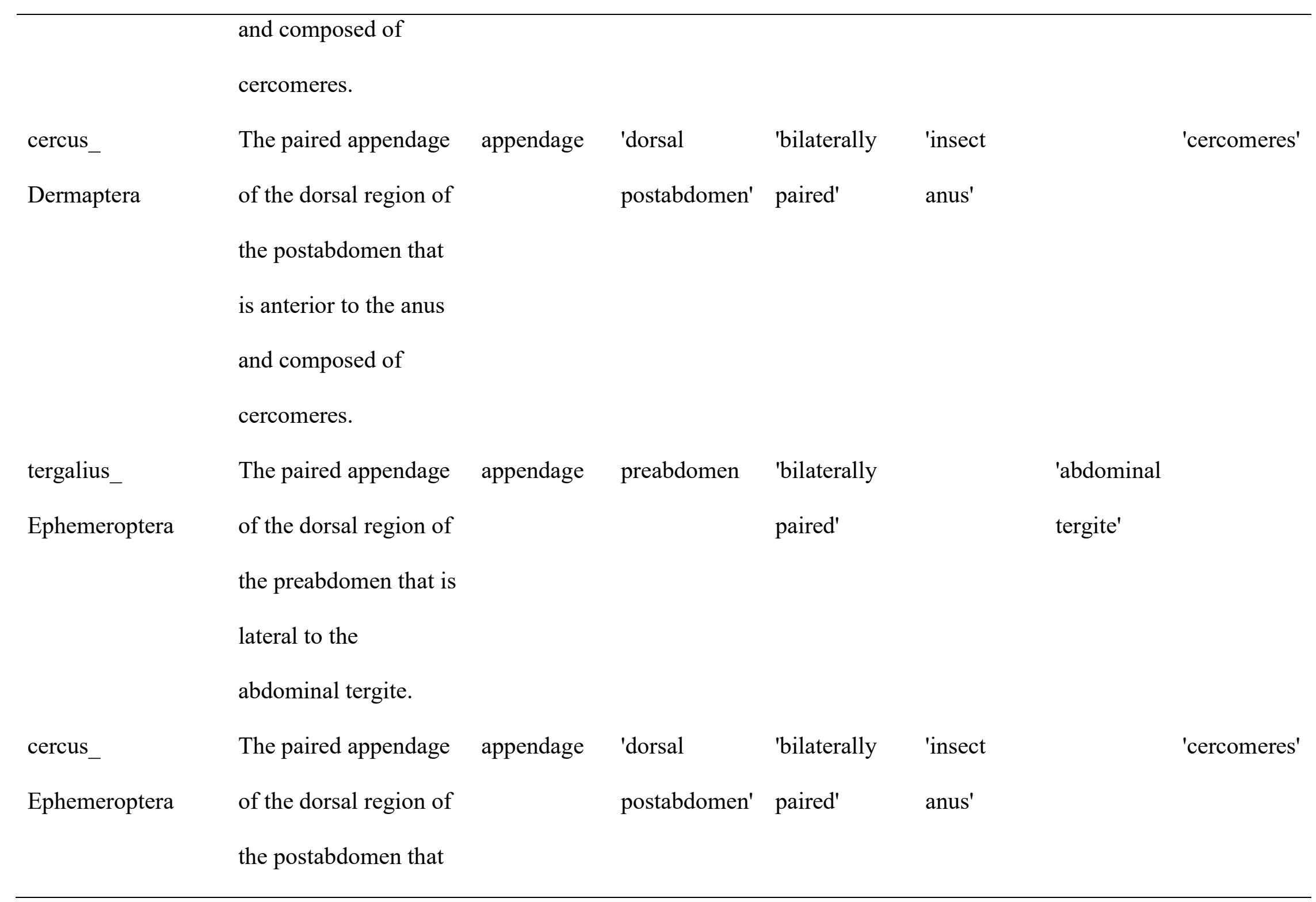




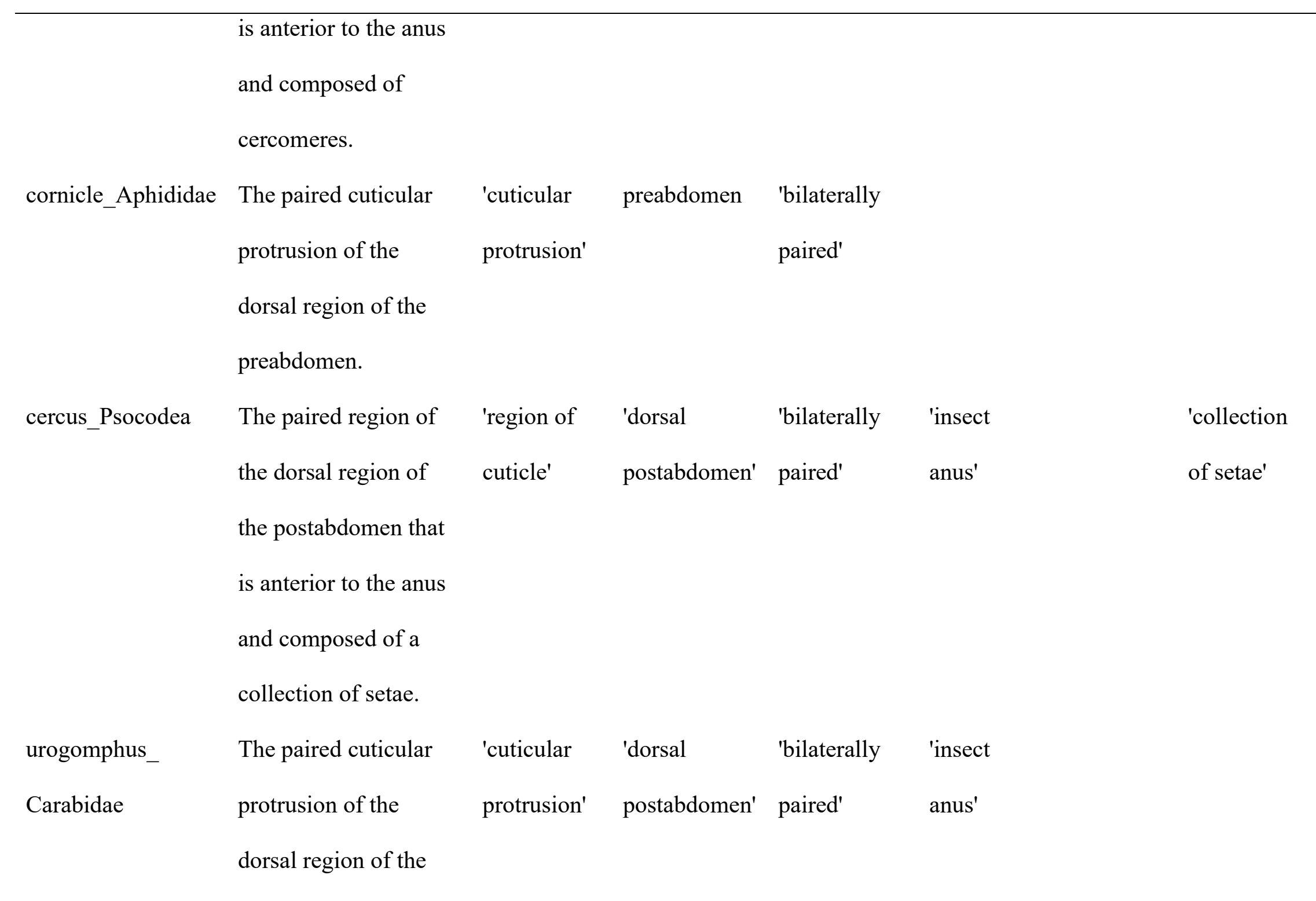


postabdomen that is

anterior to the anus.

3 
Archaeognatha

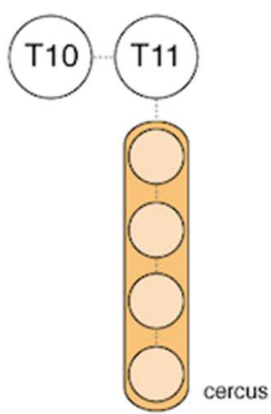

Zygentoma

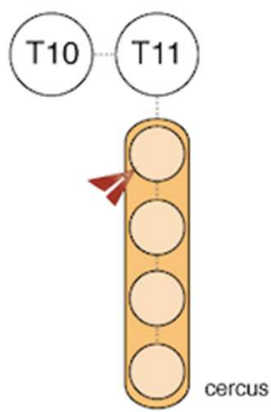

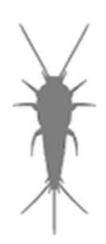

cercus
Dermaptera

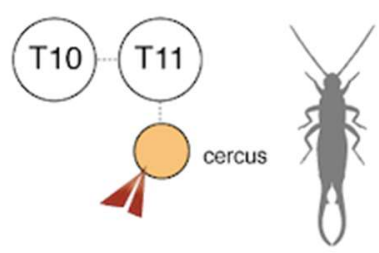

Hemiptera: Aphididae

Ephemeroptera: Baetidae (nymph)

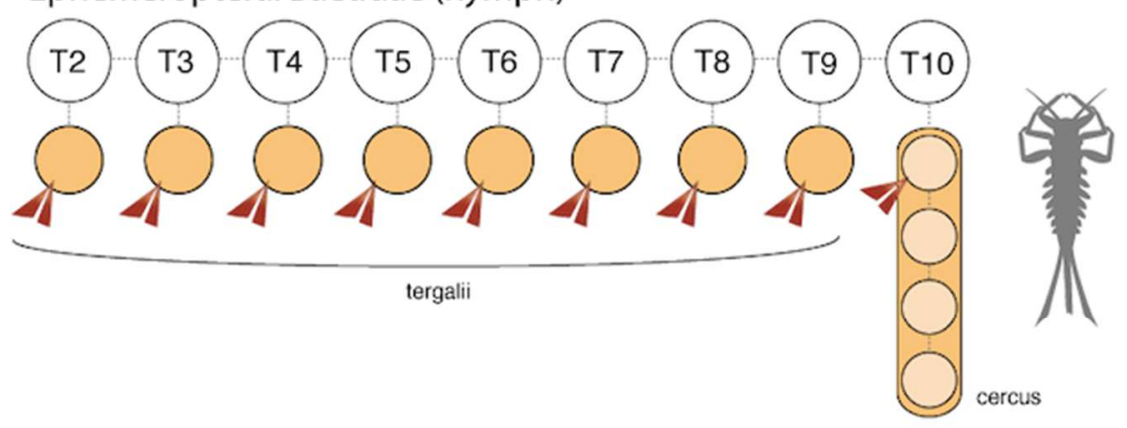

Psocodea: Psocidae

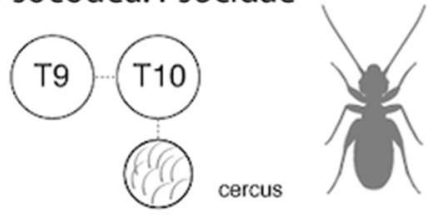

Coleoptera: Carabidae (larva)

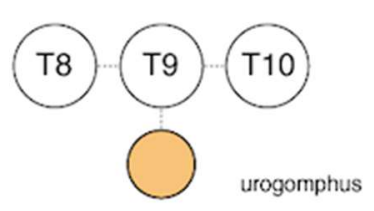

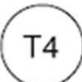
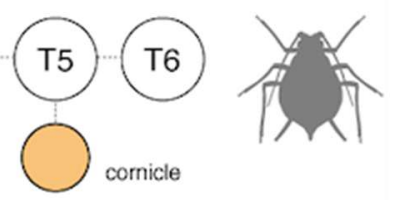

Figure 3. Schematic representation of taxon-specific definitions for paired cuticular structures of the abdominal tergites (not including structures of the genitalia) across different insect orders.

7 Textual definitions for each structure are provided in Table 3.

9 Table 4. Example DL queries and their results using the AISM.

\section{Query Resulting subclasses and individuals}

\section{Internal queries}
'cuticular protrusion' and ('part of' some
Classes: 'cuticle of insect mandible', 'cuticle
'cuticle of insect head')
of insect maxilla', 'cuticle of labial palpus' 


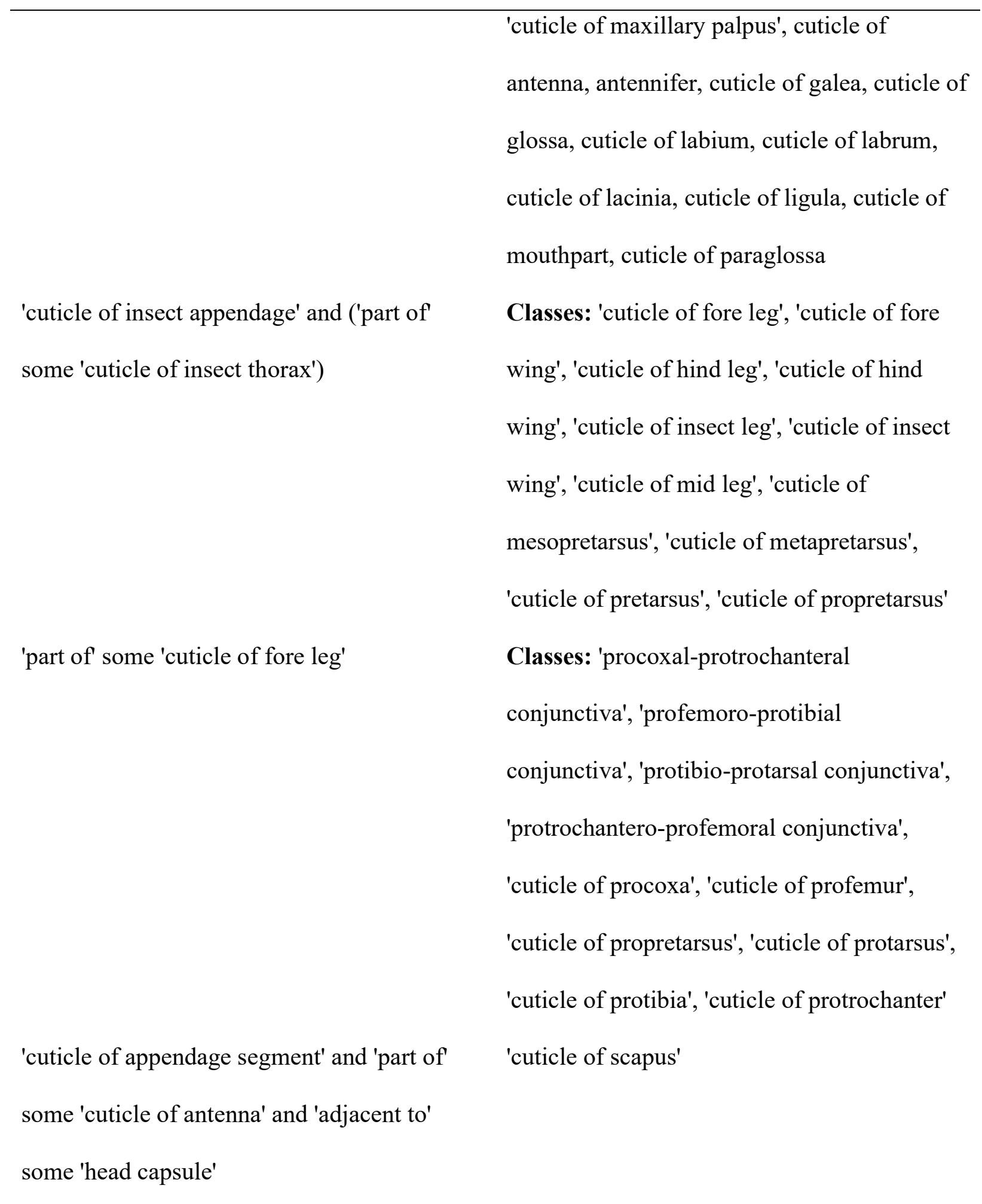

Queries for taxon-specific definitions 
'part of' some 'cuticle of insect abdomen' and 'region of cuticle' and 'has characteristic' some 'bilaterally paired'

Classes: 'cuticle of gonocoxa IX', 'cuticle of 'bilaterally paired'

\begin{tabular}{|c|c|}
\hline & cuticle of paramere \\
\hline & Instances: cercus_Archaeognatha, \\
\hline & cercus_Dermaptera, cercus_Ephemeroptera, \\
\hline & cercus_Psocodea, cercus_Zygentoma, \\
\hline & cornicle_Aphididae, \\
\hline & tergalius_Ephemeroptera, \\
\hline & urogomphus_Carabidae \\
\hline 'part of' some preabdomen and 'region of & Instances: cornicle_Aphididae, \\
\hline cuticle' and 'has characteristic' some & tergalius_Ephemeroptera \\
\hline 'bilaterally paired' & \\
\hline 'part of' some postabdomen and 'region of & Classes: 'cuticle of gonocoxa IX', 'cuticle of \\
\hline cuticle' and 'has characteristic' some & gonocoxa VIII', 'cuticle of gonostylus IX', \\
\hline 'bilaterally paired' & 'cuticle of gonostylus VIII', cuticle of cercus, \\
\hline & cuticle of paramere \\
\hline & Instances: cercus_Archaeognatha, \\
\hline & cercus_Dermaptera, cercus_Ephemeroptera, \\
\hline & cercus_Psocodea, cercus_Zygentoma, \\
\hline & urogomphus_Carabidae \\
\hline
\end{tabular}




'part of' some postabdomen and 'cuticular Classes: 'cuticle of paramere'
protrusion' and 'has characteristic' some
'bilaterally paired'

Instances: cercus_Archaeognatha, cercus_Dermaptera, cercus_Ephemeroptera, cercus_Zygentoma, urogomphus_Carabidae

'part of' some postabdomen and 'cuticular Classes: none

protrusion' and 'has characteristic' some

'bilaterally paired' and 'anterior to' some

'insect anus'

Instances: cercus_Archaeognatha, cercus_Dermaptera, cercus_Ephemeroptera, cercus_Zygentoma, urogomphus_Carabidae

'part of' some postabdomen and 'cuticle of Classes: none insect appendage' and 'has characteristic' some 'bilaterally paired' and anterior_to some 'insect anus'

Instances: cercus_Dermaptera, cercus_Ephemeroptera, cercus_Zygentoma

11 In addition, to demonstrate the interoperability of the AISM with existing ontologies, we

12 provide an example on how to describe a particular insect species phenotype. The ability to relate 13 structures across additional ontologies was also illustrated by linking structures of the AISM 
14 with the circulatory system using relationships from the Relation Ontology (RO) and terms from

15 the Ontology of Arthropod Circulatory Systems (OArCS).

\section{RESULTS AND DISCUSSION}

\section{MoDIAS: Model for Describing Insect Anatomical Structures}

MoDIAS is a descriptive model: a set of principles based on traditional approaches to

20 classify and characterize cuticular elements based on their structural properties and relative

21 position. It is proposed as a baseline to generate consistent and reproducible descriptions of

22 insect skeleto-muscular structures.

The insect endo- and exoskeleton is a continuous entity that can be considered as a single

25 anatomical structure (Klass 2008; similar to the skin of a vertebrate). Cuticular elements can be

26 defined and distinguished from each other by variations along five key properties: 1) degree of

27 flexibility (i.e., stiffness or resistance to deflection: sclerite vs. conjunctiva), 2) degree of surface

28 curvature (i.e., deviations from a flat surface: depression vs. protrusion), 3) presence of muscular

29 attachments, 4) quantity (single vs. multiple repeated elements), and 5) shape (circular,

30 elongate). The interplay of these properties determines the features of each elementary building

31 block that, together with its spatial relations and connectedness, allows for modelling of the

32 entire structural diversity of the insect skeleto-muscular system. Similar categorical sets of

33 properties have been employed in other semantic descriptive models of anatomical systems (e.g.,

34 OArCS, Ontology of Arthropod Circulatory System; Wirkner et al. 2017) to allow for better data 35 structuring. 
Based on their degree of flexibility there are two main regions of the cuticle: 1) sclerites,

38 which are relatively stiff, and 2) conjunctivae which are relatively flexible and provide mobility

39 (Klass and Matushkina 2012; Fig. 2, y axis). Even though these different regions of the cuticle

40 are often characterized by their histological properties (sclerites with thick exocuticle with

41 sclerotin vs. conjunctiva with thin exocuticle without sclerotin; Beutel et al. 2014), these are not

42 discernible without histological sections.

Regions of the cuticle are also classified by the degree of curvature of their internal and

45 external surfaces (Fig. 2, $\mathrm{x}$ axis and color bands on top right subplane), which not only provide

46 information about external components but also allows for the linking of structural variations of

47 the underlying epidermis. The external surface can be flat, convex (cuticular protrusions) or

48 concave (cuticular depressions). Cuticular protrusions, if they correspond to evaginations of the

49 cuticle (i.e., cuticular protrusion corresponding to an internal cuticular depression; Fig. 2, top left

50 subplane), can correspond to either the evagination of a single cell membrane (e.g., seta) or to the

51 evagination of a region of the single-layered outer epithelium (e.g., spurs, lobes) (Richards and

52 Richards 1979). Appendages differ from other cuticular protrusions (e.g., spurs or lobes) in that

53 they are connected to the rest of the body by somatic muscles (Fig. 2). When individual elements

54 like a carina or a seta are repeated across a region of the cuticle they generate texture on that

55 particular surface, forming sculpture or pilosity, respectively. Ring sclerites often represent

56 repetitive subdivisions of appendages that can be either musculated (appendage segments) or

57 non-musculated (meres). 
Cuticular depressions (Fig. 2, top right subplane) vary in constitution depending on the

60 orientation of the external and the internal surfaces of the cuticle: when both run in parallel, they

61 form hollow depressions (e.g., pit, sulcus; Fig. 2, top of green band); the external surface can be

62 depressed, with the internal surface flat (e.g., fovea, groove; Fig. 2, purple band); or the external

63 surface can be flat, with the internal surface depressed (e.g., ridge, apodeme; Fig. 2, blue band,

64 bottom of green band); this particular kind of cuticular depression forms strengthened areas

65 across the body, providing mechanical stability, and frequently constitute sites for muscle

66 attachment (Klass and Matushkina,2012; Beutel et al. 2014).

67

Each of these elementary building blocks with their particular features can be specifically

69 characterized by their connections and spatial relations to other elementary building blocks,

70 including positional relationships (e.g., dorsal, ventral, distal, proximal, medial, lateral),

71 connectedness (e.g., continuous with, encircled by, adjacent to) and further phenotypic

72 descriptors (color, relative size). This specific characterization results in accurate, consistent, and

73 reproducible descriptions of insect anatomical structures. If employed correctly, MoDIAS-based

74 natural language definitions should be easily translated into logical definitions and instance-

75 based semantic phenotype descriptions of individual specimens using ontologies, so that

76 information of the insect skeleto-muscular system can be accessible for machine processing.

\section{AISM: Ontology for the Anatomy of the Insect Skeleto-Muscular system}

In its version 2021-12-13, the ontology for the Anatomy of the Insect Skeleto-Muscular system (AISM) contains 1647 classes, where 532 are AISM terms, from which 384 are

81 subclasses of insect region of integument (UBERON:6007284); it uses 24 object properties and 
8220 annotation properties. All other terms have been imported from existing ontologies as part of

83 the basic imports using the Ontology Development Kit (ODK; see methods), which not only

84 brings the specified terms, but also all their hierarchically associated terms to preserve the logical

85 integrity of the ontology and maximize interoperability.

The insect integument, as a continuous structure, is composed of chitin-based cuticle

88 (UBERON:0001001); therefore, every component of this continuous structure is designated as a

89 region of cuticle (AISM:0000174), which is the parent class for all skeletal anatomical

90 structures in the AISM (Fig. 4). Interpreting the skeleto-muscular system of insects as a set of

91 consistently organized components and following the framework proposed by MoDIAS, each

92 class included in the AISM is defined logically in OWL by some combination of: (1) kind of

93 cuticular element [e.g., sclerite (AISM:0000003), conjunctiva (AISM:0000004), cuticular

94 depression (AISM:0000005), cuticular protrusion (AISM:0000008), skeletal muscle tissue

95 (UBERON:0001134), among others]; (2) location of structure in the body [e.g., part of the insect

96 thorax (AISM:0000108), anterior to the abdominal tergite I (AISM:0000021)]; (3) connected

97 structures indicated by specific relations and spatial descriptors [e.g., adjacent to posterior

98 margin (BSPO:0000672) of abdominal sternite III (AISM:0004105)]. In this way the

99 continuous nature of the insect integument is considered, making explicit statements about

100 connectivity between parts and providing positional/spatial localization for each structure. 


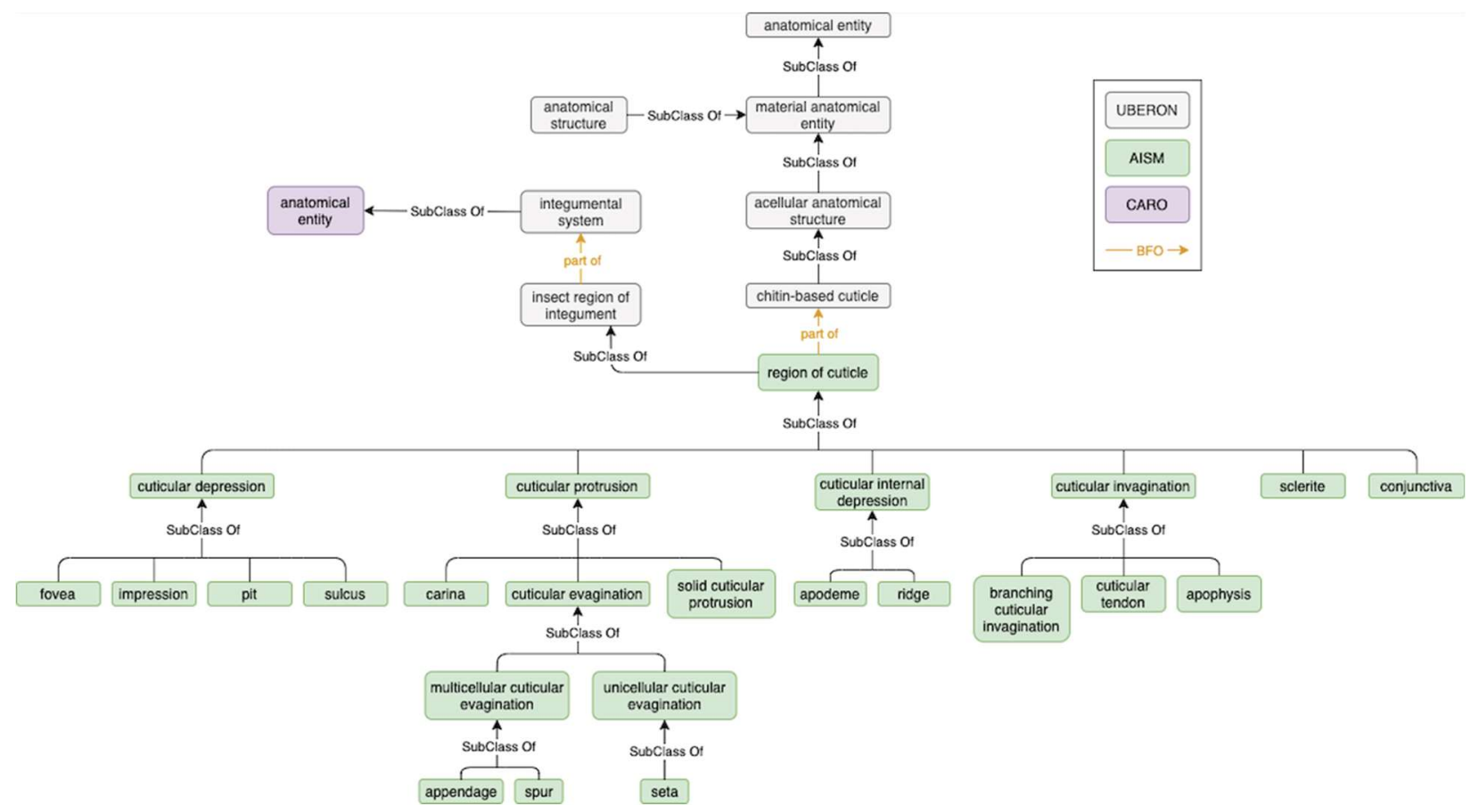

103 Figure 4. Graphic representation of high-level classes in AISM and some of their children,

104 including hierarchy and elements from other ontologies.

In addition, each class (i.e., label and descriptors) is accompanied by a set of annotation

107 properties including a natural language definition that has been created from the annotated

108 descriptors (or vice versa). These definitions include the contributor who constructed the

109 definition and the date of creation and date of modification in format year-month-day. When

110 available, references for textual definitions from the literature have been annotated on each label

111 using the annotation property sensu (AISM:0000171), which includes the full citation of the

112 reference, a DOI or link when the reference is available online, and the verbatim definition

113 provided in the text, in quotation marks (see Yoder et al. 2010). When explicitly mentioned in

114 the literature, synonyms are added using the has_exact_synonym property indicating the sensu

115 where this synonymy is proposed. 


\section{Using templates to curate and extend the AISM}

118 In order to make the AISM maximally accessible and re-usable, the AISM aims for

119 MoDIAS-based definitions that follow consistent, simple patterns. To ensure that users of the

120 AISM can also easily generate MoDIAS/AISM-compliant descriptions of anatomical structures,

121 we provide a template system for composing definitions (i.e., https://github.com/insect-

122 morphology/aism/blob/master/AISM template examples.tsv). This template system can be used

123 to provide formal descriptions of insect anatomical structures, or for extending the AISM with

124 taxon-specific terms (subclasses). Even if the templates are not directly used, they provide

125 guidance for the types of definitions that are compatible with the MoDIAS/AISM approach to

126 defining terms.

127

128 The aim of the templates is to ensure that users provide the specific type of cuticular element

129 (Fig. 2) and its appropriate location within the insect body. Users may further refine the location

130 by specifying the structure's relative location via multiple statements using relations such as

131 adjacent to, posterior to, and dorsal to. The template also includes a free text comment column

132 allowing additional information to be provided in a less formal manner. Once this detailed,

133 MoDIAS-compliant description is provided, users may also propose a commonly used term for

134 the described structure, such as 'cercus'. The advantage of this approach is that it forces users to

135 provide an accurate description of the structure's properties and location not captured by the

136 generally used term.

138 Even without additional processing, the filled-out template constitutes a shareable and

139 accessible controlled description of anatomical structures. Because the template corresponds to a 
140 standard OWL template system, it can also be used to generate new terms or instances, either for

141 the AISM, or for extending the AISM with taxon-specific terms.

142

In table 3 we present a few examples of terms to refer to different paired structures of the

144 abdomen of different insect taxa. For instance, the cercus of Archaeognatha, defined as the

145 paired protrusion of the dorsal region of the postabdomen that is anterior to the anus and

146 composed of cercomeres is indicated in the template specifying its type of cuticular element

147 (cuticular protrusion, AISM:0000008), its location (dorsal postabdomen, AISM:0000523), its

148 laterality (bilaterally paired, PATO:0040024), its position regarding other structures (anterior to

149 [BSPO:0000096] insect anus [AISM:0004197]), and its composition (cuticle of cercomeres,

150 AISM:0004199). As examples are specified, OWL reasoning can be used to provide a list of

151 candidate terms in AISM that conform to the definition.

The current implementation of the templates relies on users following the specification.

154 In the future, we plan to make use of the CEDAR template system

155 (https://more.metadatacenter.org/tools-training/cedar-template-tools/\#design-template) to

156 provide auto-completion and constraints on column content, guiding and constraining users to

157 ensure the correct types of terms are added in each column. We also plan to integrate a term

158 suggestion option to avoid replication and detect potential synonyms.

160 Reasoning with the AISM

161 Each of the queries tested returned the expected outcomes in terms of subclasses and

162 individuals (Table 4). Across Insecta, abdominal protrusions are highly variable in position, 
163 shape, and components, and in many cases the morphological interpretations of these structures

164 and their features have been problematic over time. The terms and broad definitions presented in

165 the AISM have the capability to incorporate the broad variation presented in our example taxa.

166 By adding subclasses and relationships to AISM terms it is possible to characterize taxon-

167 specific structures. For instance, the cornicles of Aphididae (Hemiptera) are paired cuticular

168 protrusions located on the dorsal surface of the abdominal tergite 5 (sometimes abdominal tergite

169 6); the existing terms and definitions incorporated in the AISM allow for accommodating all

170 these details into a definition for cornicle in a potential Hemiptera-specific ontology. Similarly,

171 the tergalii of Ephemeroptera, paired appendages of the preabdomen located on the lateral region

172 of the abdominal tergites (Kluge 2004) can be easily defined, and the particular abdominal

173 tergites where the tergalii are present could be specified.

174

The different kinds of cerci present in our example taxa were also easily characterized, as

176 they follow the generalized definition proposed in the AISM (the bilaterally paired region of the

177 cuticle of the dorsal region of the postabdomen, that is anterior to the anus): in Dermaptera the

178 cercus was characterized as an appendage (with muscular attachment) and composed of a single

179 cercomere (Fig. 3). In Psocodea it was defined as a region of the cuticle that is anterior to the

180 anus and bears a collection of setae (Fig. 3); we followed the definition presented by Yoshizawa

181 (2005), even though the definition of this particular surface (anterior to the anus) in Psocodea as

182 cercus has been contentious. In Zygentoma and Ephemeroptera the cercus was characterized as a

183 paired appendage (with muscular attachment) of the dorsal region of the postabdomen,

184 composed of cercomeres, whereas in Archaeognatha, the cercus is a cuticular protrusion (without

185 muscular attachment) of the dorsal region of the postabdomen, composed of cercomeres. Our 
186 queries also returned the Coleoptera urogomphus as a cercus, as this undivided cuticular

187 protrusion satisfies the requirements of the AISM definition for cercus. This demonstrates the

188 power of the AISM's homology free approach, as these urogomphi are structurally equivalent to

189 cercus, but not homologous, a similarity that would be obscured if we only relied in homology-

190 biased terminology. On the other hand, the same query did not recover the cornicles, as these are

191 paired projections of the abdomen, but located in a different abdominal region. The amount of

192 detail incorporated into each definition will depend on the intended use of the ontology. Indeed,

193 the number and sequence of cercomeres can be specified, along with the presence of setae,

194 scales, or other relevant features.

195

196 Describing phenotypes with the AISM

197 The AISM has been conceived as the backbone ontology for taxon-specific ontologies.

198 The broadly applicable terms it contains are intended as superclasses for terms defining more

199 taxon-specific anatomical structures. For instance, in an AISM-based Coleoptera-specific

200 ontology, the class elytron would be created as a subclass of the class fore wing

201 (AISM:0000037), adding descriptors related to specific properties of elytron, such as subclass of

202 the class sclerite (AISM:0000003). Similarly, in an AISM-based Diptera-specific ontology the

203 class haltere would be a subclass of the class hind wing (AISM:0000038).

204

205

In general, for describing specific insect phenotypes with the AISM, a series of Entity-

206 Quality statements can be used (e.g., Washington et al. 2009), taking advantage of the high

207 interoperability of the AISM and the broad range of available existing ontologies including those

208 for phenotype (PATO: Phenotype And Trait Ontology), taxonomy (NCBITaxon: National Center 
209 for Biotechnology Information organismal classification), spatial relationships (BSPO:

210 Biological Spatial Ontology), among others. The template system proposed here can

211 accommodate additional descriptors and relationships to better define structures within the

212 AISM.

213

214 It is possible to represent phenotypes like a yellow profemur on a chalcid wasp:

215 [(Chalcididae [NCBITaxon:92425] AND has part [BFO:0000051] some profemur

216 [AISM:0000070]) AND (has characteristic [RO:0000053] some yellow [PATO:0000324])].

217 Using the template system, an additional column for color would be needed.

In our exercise linking AISM to OArCS, no new terms were required, just additional

220 linkages between existing terms and relationships, for example, the alary muscle

221 (OARCS:0000151) is attached to (RO:0002371) both the heart (OARCS:0000253) and the

222 abdominal tergite (AISM:0004057). The term alary muscle would be imported using the ODK,

223 bringing the necessary hierarchically linked terms and properties to be able to construct the

224 logically appropriate axioms.

Taxon-specific ontologies can be linked to specialized taxonomic ontologies if those

227 were available (e.g., see Stucky 2019). An example of taxonomic ontologies is the Vertebrate

228 Taxonomy Ontology (VTO; Midford et al. 2013), which provides a comprehensive taxonomic

229 hierarchy for vertebrates. It incorporates classes from the Taxonomic rank vocabulary

230 (http://www.obofoundry.org/ontology/ncbitaxon.html) and the NCBI organismal classification 
231 (National Center for Biotechnology Information;

232 http://www.obofoundry.org/ontology/taxrank.html).

233

234

All these approaches to phenotypic descriptions can be implemented using and extending

235 the proposed template system. Furthermore, integration between the AISM and existing

236 ontologies like Uberon, the Drosophila Anatomy Ontology (FBBT; Costa et al. 2013), and the

237 Hymenoptera Anatomy Ontology (HAO; Yoder et al. 2010) can be improved over time by

238 adding cross-reference annotations to each shared term.

239

240 Taxonomy, morphology, and evo-devo: AISM on different granularity levels

241 Similar surface modifications of the insect skeleto-muscular system can correspond with

242 cardinally different epithelial modifications: multicellular invaginations/evaginations of the

243 epidermal cell layer (e.g., cuticular depressions, spurs, pits, and appendages),

244 invaginations/evaginations of a single cell membrane (e.g., cuticular component of sensilla),

245 changes in the thickness of the cuticle (i.e., modifications that do not correspond to any epithelial

246 fold, e.g., impression, acantha or carina), and in some cases the combination of these categories.

Changes in the geometry of the epithelial sheet resulting in invaginations and

249 evaginations are governed by genes that define changes in the shape of epidermal cells or

250 regulate cell proliferation (Zartman and Shvartsman 2010; Hannezo et al. 2014; Gotoh et al.

251 2021) while genes involved in the reorganization of the cytoskeleton are governing similar

252 geometrical changes of the membrane of a single cell (Lees and Waddington 1942; Bitan et al.

253 2012; Djokic et al. 2020). A third set of genes are involved in surface characteristics that are 
254 related to cuticle thickness, which are related to processes regulating cuticle deposition (Adler

255 2017; Jan et al. 2017; Tajiri, 2017; Zhao et al. 2017). These processes are also separated in time

256 and space; evaginations and invaginations happen during the last larval and early pupal stages,

257 while cuticle deposition starts in the late pupal stage (Andersen 2012).

It is evident that differentiating these superficially similar structures would be key to

260 accurately understanding phenotypic diversity and morphological evolution. However, the

261 differences between practical approaches to anatomy across different knowledge domains

262 represent a huge communication gap that hinders progress towards a more integrative view of

263 anatomy (Richards and Richards 1979): (1) morphology aims to interpret the structural identity

264 and connectivity of anatomical structures (Snodgrass 1951); it uses dissections and section-based

265 methods ranging from histology to $\mu-\mathrm{CT}$, and usually focuses on a handful of specimens in each

266 study; (2) taxonomy focuses for the most part on externally visible structures with diagnostic

267 value; each study can involve thousands of specimens in a comparative framework; (3) evo-devo

268 studies gene expression on developing structures; the taxonomic breadth is usually limited to

269 model organisms that are reared under laboratory conditions.

271 These knowledge domains refer to anatomy at different granularity levels and from

272 different frames of reference (Vogt 2019) across different shared themes (i.e., taxonomic,

273 individual count, developmental stage), which causes interoperability problems and

274 misunderstanding among disciplines, due to concept shifting of anatomical entities. The AISM

275 provides a bridge, in the form of a controlled vocabulary, to facilitate communication, by using

276 an interconnected hierarchy of superficial cuticular elements (anatomical surfaces) and the 
277 hierarchy of deeper structures that reveal developmental and structural properties of the single-

278 layered outer epithelium. The AISM provides an opportunity to link insect phenotypes to

279 genotypes across developmental stages and taxonomic groups via the Gene Ontology (Ashburner

280 2000), and to metabolic processes via the Protein Ontology (PRO; Natale et al. 2017) and the

281 ontology for Chemical Entities of Biological Interest (CHEBI; Hastings et al. 2015).

282

283 Homology and the AISM

284 Evolutionary homology is a central concept in biology, whereby structural similarity has 285 evolved through shared ancestry in different taxa (Minelli and Fusco 2013; Wagner 2014). The 286 definitions of the classes included in the AISM are merely descriptive in anatomical terms and 287 do not constitute homology statements, although they may serve to assess the primary criteria of 288 position and similarity (see comparative homology, units of comparison; Vogt 2017), so that 289 instead of asking whether the cercus in Archaeognatha is homologous to the cercus in Psocodea, 290 we can ask if a multisegmented appendage on the 11th tergite in one group is homologous with a 291 setose patch on the 10th tergite of the other (see our examples for abdominal cuticular

292 protrusions, Table 3). There are data models such as the one proposed by Mabee et al. (2020), 293 where homology relationships can be logically formalized between anatomical structures of 294 different taxonomic units. This approach requires elements from anatomy ontologies, taxonomic 295 ontologies, and the Evidence and Conclusion Ontology (ECO, Chibucos et al. 2014). Under this 296 scenario, the AISM would serve as one of the components required to assess homology 297 statements across different taxonomic groups of insects. 
Nomenclatural rules require that the establishment of an animal taxon new to science "be

301 accompanied by a description or definition that states in words characters that are purported to

302 differentiate the taxon" or "by a bibliographic reference to such a published statement" (Article

303 13.1 in ICZN 1999). Taxonomists have described over one million species of insects worldwide

304 (Stork 2018). These descriptions constitute vast amounts of information that could be efficiently

305 mined, compared, interpreted, and analyzed, just like any large molecular dataset nowadays.

306 However, these phenotypic descriptions are presented in non-standard natural language form,

307 and are therefore, inaccessible for machine interpretation (Balhoff et al. 2010). Ontologies and

308 knowledge graphs offer a system to represent entire knowledge domains in an organized,

309 standardized, consistent, and logical manner, so that information can be processed and quality-

310 checked by computers (Arp et al. 2015).

There are informatic tools that allow data extraction from the literature, based on XML

313 markup, which have been used primarily for extracting taxonomic information from PDF files

314 (Penev et al. 2011). For instance, GoldenGATE-Imagine (Sautter et al. 2007;

315 https://github.com/plazi/GoldenGATE-Imagine), which is used by Plazi (http://plazi.org). There

316 are also tools that use ontologies for annotating anatomical, phenotypic, and taxonomic data

317 (Phenex; Balhoff et al. 2010). Lücking et al. (2021) provide an overview of methods for semantic

318 annotation of bibliographic records and introduce a system to use multiple annotations for terms;

319 the authors also introduce the BIOfid-portal (https://www.biofid.de/en/search/), which is an on-

320 line tool for accessing the semantics of biodiversity texts in German. The annotation method

321 proposed by Lücking et al. (2021) is partly based on the MATTER conceptual framework for 
322 annotations (Model, Annotate, Train and Test, Evaluate, and Revise; Pustejovsky \& Stubbs 323 2012).

324 The AISM provides the key to annotate phenotypic information for insects, extracted

325 from the literature. Combining or expanding these and similar informatic tools can generate

326 large-scale phenotypic datasets, unlocking multiple avenues of research including, among others,

327 genotype to phenotype associations, evo-devo studies, and the use of Artificial Intelligence and

328 ontological inference (Jackson et al. 2018) to analyze morphological evolution across insects.

329 Phenotypic data generated with the aid of the AISM would greatly contribute to increase links in

330 the Biodiversity Knowledge Graph (Page 2013).

332 Availability

333 The AISM is available on GitHub at https://github.com/insect-morphology/aism (Girón et

334 al. 2021a) as well as on the OBO Foundry at http://www.obofoundry.org/ontology/aism.html.

335 The GitHub repository is open for collaborative editing. We provide a manual on how to edit the

336 AISM (https://github.com/insect-morphology/Manual; (Girón et al. 2021b), including the use of

337 templates, and how to use the AISM as the starting point for developing taxon-specific

338 ontologies.

340 CONCLUSION

341 Here we provided a Model for Describing Insect Anatomical Structures (MoDIAS) that

342 incorporates structural properties and positional relationships to define anatomical structures of

343 insects, independent of developmental stage, homology assumptions, or taxonomic group.

344 Following the set of principles established by MoDIAS, we created the first universally 
345 applicable anatomy ontology for insects, the Ontology for the Anatomy of the Insect Skeleto-

346 Muscular system (AISM). The AISM provides a basic backbone of generalized and

347 unambiguously labeled terms for the anatomy of the skeleto-muscular system of insects. Each

348 term is accompanied by natural language definitions translated into sets of subclass of

349 descriptors to provide logical definitions in the ontology. Built using the Ontology Development

350 Kit, which is a free, open source, and OBO Foundry-supported system, the AISM is

351 interoperable with existing ontologies in the biological sciences, open for editing and refinement,

352 and extensible to tackle taxon-specific ontologies.

The AISM opens new opportunities for phenomic-scale research in biology by providing 355 computer-parsable formalization and a controlled vocabulary for insect anatomy. The potential 356 application of AISM spans all biological domains, including phenotype comparison and 357 description, and ontology-informed phylogenetic methods (Tarasov 2019).

\section{FUNDING}

360 Funding was provided by the NSF ARTS Program (DEB \#1523605 and DEB \#2009247

361 to Aaron Smith). Sergei Tarasov was supported by the Academy of Finland grant: 339576, and

362 three-year grant from the University of Helsinki. Markus Koch was supported by grants of the

363 German Research Foundation (DFG-project MO 412/54-1) for the Specialized Information

364 Service Biodiversity Research (BIOfid). Brendon Boudinot was supported by the Alexander von

365 Humboldt Stiftung (Germany). 


\section{REFERENCES}

369

370 Adachi, H., Matsuda, K., Nishida, K., Hanson, P., Kondo, S. \& Gotoh, H. (2020) Structure and

371 development of the complex helmet of treehoppers (Insecta: Hemiptera: Membracidae).

372

Zoological Letters 6, 3. https://doi.org/10.1186/s40851-020-00155-7

373

374 Adler, P.N. (2017) Gene expression and morphogenesis during the deposition of Drosophila

375 wing cuticle. Fly 11, 194-199. https://doi.org/10.1080/19336934.2017.1295188

376

377 Andersen, S.O. (2012) Cuticular sclerotization and tanning. In: Insect molecular biology and 378 biochemistry. Elsevier, pp. 167-192.

379

380 Arp, R., Smith, B. \& Spear, A.D. (2015) Building Ontologies with Basic Formal Ontology. MIT

381 Press, Cambridge, MA, USA, 248 pp.

382

383 Ashburner, M., Ball, C.A., Blake, J.A., Botstein, D., Butler, H., Cherry, J.M., Davis, A.P.,

384 Dolinski, K., Dwight, S.S., Eppig, J.T., Harris, M.A., Hill, D.P., Issel-Tarver, L., Kasarskis,

385 A., Lewis, S., Matese, J.C., Richardson, J.E., Ringwald, M., Rubin, G.M. \& Sherlock, G.

386 (2000) Gene Ontology: tool for the unification of biology. Nature Genetics 25, 25-29.

$387 \quad$ https://doi.org/10.1038/75556

388 
Balhoff, J.P., Dahdul, W.M., Kothari, C.R., Lapp, H., Lundberg, J.G., Mabee, P., Midford, P.E., Westerfield, M. \& Vision, T.J. (2010) Phenex: Ontological Annotation of Phenotypic Diversity. PLOS ONE 5, e10500. https://doi.org/10.1371/journal.pone.0010500

Balhoff, J.P., Mikó, I., Yoder, M.J., Mullins, P.L. \& Deans, A.R. (2013) A Semantic Model for Species Description Applied to the Ensign Wasps (Hymenoptera: Evaniidae) of New Caledonia. Systematic Biology 62, 639-659. https://doi.org/10.1093/sysbio/syt028

Beutel, R.G., Friedrich, F., Yang, X.-K. \& Ge, S.-Q. (2014) Insect Morphology and Phylogeny Insect morphology and phylogeny: a textbook for students of entomology. De Gruyter. https://doi.org/10.1515/9783110264043

Bitan, A., Rosenbaum, I. \& Abdu, U. (2012) Stable and dynamic microtubules coordinately determine and maintain Drosophila bristle shape. Development 139, 1987-1996. $\underline{\text { https://doi.org/10.1242/dev.076893 }}$

Bitsch, C. \& Bitsch, J. (2002) The endoskeletal structures in arthropods: cytology, morphology and evolution. Arthropod Structure \& Development 30, 159-177. https://doi.org/10.1016/S1467-8039(01)00032-9

Blumer, M.J.F. (2021) Bone tissue and histological and molecular events during development of the long bones. Annals of Anatomy - Anatomischer Anzeiger 235, 151704. $\underline{\text { https://doi.org/10.1016/j.aanat.2021.151704 }}$ 
413 Bolshoy, A. \& Lacková, L. (2021) Illusions of Linguistics and Illusions of Modern Synthesis:

414 Two Parallel Stories. Biosemiotics 14, 115-119. https://doi.org/10.1007/s12304-021-09429-9

415

416 Chibucos, M.C., Mungall, C.J., Balakrishnan, R., Christie, K.R., Huntley, R.P., White, O., Blake, 417 J.A., Lewis, S.E. \& Giglio, M. (2014) Standardized description of scientific evidence using 418 the Evidence Ontology (ECO). Database 2014. https://doi.org/10.1093/database/bau075

420 Costa, M., Reeve, S., Grumbling, G. \& Osumi-Sutherland, D. (2013) The Drosophila anatomy 421 ontology. Journal of Biomedical Semantics 4, 32. https://doi.org/10.1186/2041-1480-4-32

423 Dahdul, W.M., Cui, H., Mabee, P.M., Mungall, C.J., Osumi-Sutherland, D., Walls, R.L. \& 424 Haendel, M.A. (2014) Nose to tail, roots to shoots: spatial descriptors for phenotypic 425 diversity in the Biological Spatial Ontology. Journal of Biomedical Semantics 5, 34.

$426 \quad$ https://doi.org/10.1186/2041-1480-5-34

427

428 Dahdul W., Manda P., Cui H., Balhoff J.P., Dececchi T.A., Ibrahim N., Lapp H., Vision T., 429 Mabee P.M. (2018) Annotation of phenotypes using ontologies: a gold standard for the $430 \quad$ training and evaluation of natural language processing systems. Database, bay110: $17 \mathrm{pp}$. $431 \quad$ https://doi.org/10.1093/database/bay110

433 de Oliveira, V.P. \& Cruz-Landim, C. (2003) Morphology and function of insect fat body cells: A 434 review. Biociências 11, 195-205. 
436 Deans, A.R., Mikó, I., Wipfler, B. \& Friedrich, F. (2012a) Evolutionary phenomics and the

437 emerging enlightenment of arthropod systematics. Invertebrate Systematics 26, 323-330.

$438 \quad$ https://doi.org/10.1071/IS12063

439

440 Deans, A.R., Yoder, M.J., Balhoff, J.P. (2012) Time to change how we describe biodiversity.

441 Trends in Ecology \& Evolution 27, 78-84. https://doi.org/10.1016/j.tree.2011.11.007

443 Deans AR, Lewis SE, Huala E, Anzaldo SS, Ashburner M, Balhoff JP, Blackburn DC, Blake JA, 444 Burleigh JG, Chanet B, Cooper LD, Courtot M, Csösz S, Cui H, Dahdul W, Das S, Dececchi 445 TA, Dettai A, Diogo R, Druzinsky RE, Dumontier M, Franz NM, Friedrich F, Gkoutos GV, 446 Haendel M, Harmon LJ, Hayamizu TF, He Y, Hines HM, Ibrahim N, Jackson LM, Jaiswal P, 447 James-Zorn C, Köhler S, Lecointre G, Lapp H, Lawrence CJ, Novère NL, Lundberg JG, 448 Macklin J, Mast AR, Midford PE, Mikó I, Mungall CJ, Oellrich A, Osumi-Sutherland D, 449 Parkinson H, Ramírez MJ, Richter S, Robinson PN, Ruttenberg A, Schulz KS, Segerdell E, 450 Seltmann KC, Sharkey MJ, Smith AD, Smith B, Specht CD, Squires RB, Thacker RW, 451 Thessen A, Fernandez-Triana J, Vihinen M, Vize PD, Vogt L, Wall CE, Walls RL, 452 Westerfeld M, Wharton RA, Wirkner CS, Woolley JB, Yoder MJ, Zorn AM, Mabee P (2015) 453 Finding Our Way through Phenotypes. PLOS Biology 13: e1002033.

$454 \quad$ https://doi.org/10.1371/journal.pbio.1002033 
456 Denk-Lobnig, M. \& Martin, A.C. (2020) Divergent and combinatorial mechanical strategies that

457 promote epithelial folding during morphogenesis. Current Opinion in Genetics \&

458 Development 63, 24-29. https://doi.org/10.1016/j.gde.2020.02.014

459

460 Djokic, S., Bakhrat, A., Tsurim, I., Urakova, N., Rasgon, J.L. \& Abdu, U. (2020) Actin bundles

461 play a different role in shaping scales compared to bristles in the mosquito Aedes aegypti.

462 Scientific Reports 10, 14885. https://doi.org/10.1038/s41598-020-71911-0

464 Dönitz, J., Grossmann, D., Schild, I., Schmitt-Engel, C., Bradler, S., Prpic, N.-M. \& Bucher, G. (2013) TrOn: An Anatomical Ontology for the Beetle Tribolium castaneum. PLOS ONE 8,

467

Girón, J.C., Mikó, I., González Montaña, L.A., Tarasov, S. \& Matentzoglu, N. (2021) Ontology e70695. https://doi.org/10.1371/journal.pone.0070695 for the Anatomy of the Insect Skeleto-Muscular System (AISM), v2021-09-07.

471

472 Girón, J.C., González Montaña, L.A. \& Mikó, I. (2021b) How to create AISM-based insect 473 anatomy ontologies using the Ontology Development Kit (ODK) and edit them in Protégé. https://doi.org/10.5281/zenodo.4642556

476 Gkoutos, G.V., Green, E.C., Mallon, A.-M., Hancock, J.M. \& Davidson, D. (2004) Using 477 ontologies to describe mouse phenotypes. Genome Biology 6, R8. https://doi.org/10.1186/gb$478 \quad \underline{2004-6-1-r 8}$ 
480 González-Montaña, L. (2021a). Collembola Anatomy Ontology. GitHub repository.

481 https://github.com/luis-gonzalez-m/Collembola. https://doi.org/10.5281/zenodo.4660386

482

483 González-Montaña, L. (2021b) Semantic-based methods for morphological descriptions: An

484 applied example for Neotropical species of genus Lepidocyrtus Bourlet, 1839 (Collembola:

485 Entomobryidae). Biosystematics and Ecology 1, e71620: 16 pp.

$486 \quad$ https://doi.org/10.1553/biosystecol.1.e71620

487

488 Gotoh, H., Adachi, H., Matsuda, K. \& Lavine, L.C. (2021) Epithelial folding determines the final 489 shape of beetle horns. Current Opinion in Genetics \& Development 69, 122-128. https://doi.org/10.1016/j.gde.2021.03.003

491

492 Haendel, M.A., Neuhaus, F., Osumi-Sutherland, D., Mabee, P.M., Mejino, J.L.V., Mungall, C.J.

493 \& Smith, B. (2008) CARO - The Common Anatomy Reference Ontology. In: A. Burger, D.

494 Davidson, and R. Baldock (Eds), Anatomy Ontologies for Bioinformatics: Principles and

495 Practice. Computational Biology. Springer, London, pp. 327-349.

496 https://doi.org/10.1007/978-1-84628-885-2 16

497

498 Hall, B.K. (1975) Evolutionary Consequences of Skeletal Differentiation. American Zoologist

$499 \quad 15,329-350 . \underline{\text { https://doi.org/10.1093/icb/15.2.329 }}$

500 
501 Hannezo, E., Prost, J. \& Joanny, J.-F. (2014) Theory of epithelial sheet morphology in three

502 dimensions. Proceedings of the National Academy of Sciences 111, 27-32.

503 https://doi.org/10.1073/pnas.1312076111

504

505

Hastings, J., Owen, G, Dekker, A., Ennis, M., Kale, N., Muthukrishnan, V., Turner, S., Swainston, N., Mendes, P., Steinbeck, C. (2016) ChEBI in 2016: Improved services and an expanding collection of metabolites. Nucleic Acids Research, 44: D1214-D1219.

509

ICZN (1999) International Code of Zoological Nomenclature. Fourth edition, adopted by the $\underline{\text { https://doi.org/10.1093/nar/gkv1031 }}$

514 Iyer, J., Wang, Q., Le, T., Pizzo, L., Grönke, S., Ambegaokar, S.S., Imai, Y., Srivastava, A.,

515 Troisí, B.L., Mardon, G., Artero, R., Jackson, G.R., Isaacs, A.M., Partridge, L., Lu, B., 516 Kumar, J.P. \& Girirajan, S. (2016) Quantitative Assessment of Eye Phenotypes for 517 Functional Genetic Studies Using Drosophila melanogaster. G3 Genes|Genomes $\mid$ Genetics 6, 1427-1437. https://doi.org/10.1534/g3.116.027060

520 Jackson L.M., Fernando P.C., Hanscom J.S., Balhoff J.P., Mabee P.M. (2018) Automated

521 Integration of Trees and Traits: A Case Study Using Paired Fin Loss Across Teleost Fishes.

522 Systematic Biology, 67: 559-575. https://doi.org/10.1093/sysbio/syx098 
524 Jackson, R.C., Balhoff, J.P., Douglass, E., Harris, N.L., Mungall, C.J. \& Overton, J.A. (2019)

525 ROBOT: A Tool for Automating Ontology Workflows. BMC Bioinformatics 20, 407.

$526 \quad$ https://doi.org/10.1186/s12859-019-3002-3

527

528 Jan, S., Liu, S., Hafeez, M., Zhang, X., Dawar, F.U., Guo, J., Gao, C. \& Wang, M. (2017)

529 Isolation and functional identification of three cuticle protein genes during metamorphosis of

530 the beet armyworm, Spodoptera exigua. Scientific Reports 7, 16061.

$531 \quad$ https://doi.org/10.1038/s41598-017-16435-w

532

533 Klass, K.-D. (2008) The female abdomen of ovipositor-bearing odonata (insecta: Pterygota).

534 Arthropod Systematics \& Phylogeny 66, 45-142. https://www.zobodat.at/pdf/Arthropod-

$535 \quad$ Systematics-Phylogeny 66 0045-0142.pdf

536

537 Klass, K.-D. \& Matushkina, N.A. (2012) The exoskeleton of the female genitalic region in

538 Petrobiellus takunagae (Insecta: Archaeognatha): Insect-wide terminology, homologies, and

539 functional interpretations. Arthropod Structure \& Development 41, 575-591.

$540 \quad$ https://doi.org/10.1016/j.asd.2012.06.003

541

542 Kluge, N. (2004) The phylogenetic system of Ephemeroptera. Springer Science \& Business

543 Media, 442 pp. https://link.springer.com/book/10.1007/978-94-007-0872-3 
545 Lees, A.D. \& Waddington, C. (1942) The development of the bristles in normal and some mutant 546 types of Drosophila melanogaster. Proceedings of the Royal Society of London. Series B -

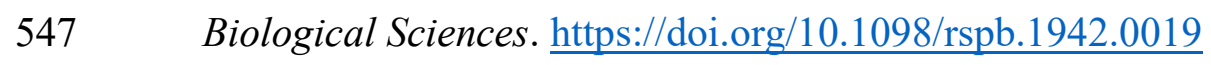

549 Loesel, R., Wolf, H., Kenning, M., Harzsch, S. \& Sombke, A. (2013) Architectural Principles 550 and Evolution of the Arthropod Central Nervous System. In: A. Minelli, G. Boxshall, and G. 551 Fusco (Eds), Arthropod Biology and Evolution: Molecules, Development, Morphology. 552 Springer, Berlin, Heidelberg, pp. 299-342.

554 Lücking, A., Driller, C., Stoeckel, M., Abrami, G., Pachzelt, A. \& Mehler, A. (2021) Multiple 555 annotation for biodiversity: developing an annotation framework among biology, linguistics 556 and text technology. Language Resources and Evaluation. https://doi.org/10.1007/s10579$557 \quad 021-09553-5$

559 Mabee, P.M., Balhoff, J.P., Dahdul, W.M., Lapp, H., Mungall, C.J. \& Vision, T.J. (2020) A 560 Logical Model of Homology for Comparative Biology. Systematic Biology 69, 345-362. https://doi.org/10.1093/sysbio/syz067

563 Matentzoglu, N., Mungall, C., Osumi-Sutherland, D., Balhoff, J., Douglass, E., Vasilevsky, N., 564 Larralde, M., Buttigieg, P.L., bjonnh, Duncan, B., Laporte, M.-A., white, marijane, Overton, 565 J.A., Seager, J., Calder, J., Blumberg, K., Lee, R. \& Robb, S. (2021) INCATools/ontology566 development-kit: 2021-04-04 release. Zenodo. https://doi.org/10.5281/zenodo.4662067 
568 Midford, P.E., Dececchi, T.A., Balhoff, J.P., Dahdul, W.M., Ibrahim, N., Lapp, H., Lundberg,

569 J.G., Mabee, P.M., Sereno, P.C., Westerfield, M., Vision, T.J. \& Blackburn, D.C. (2013) The

570 vertebrate taxonomy ontology: a framework for reasoning across model organism and

571 species phenotypes. Journal of Biomedical Semantics 4, 34. https://doi.org/10.1186/2041-

$572 \quad \underline{1480-4-34}$

573

574 Minelli, A. \& Fusco, G. (2013) Homology. In: K. Kampourakis (Ed), The Philosophy of Biology:

575 A Companion for Educators. History, Philosophy and Theory of the Life Sciences. Springer

576 Netherlands, Dordrecht, pp. 289-322. https://doi.org/10.1007/978-94-007-6537-5 15

578 Monteiro, E.C., Tamaki, F.K., Terra, W.R. \& Ribeiro, A.F. (2014) The digestive system of the

579 “stick bug” Cladomorphus phyllinus (Phasmida, Phasmatidae): A morphological,

580 physiological and biochemical analysis. Arthropod Structure \& Development 43, 123-134.

$581 \quad$ https://doi.org/10.1016/j.asd.2013.11.005

582

583 Mungall, C., Osumi-Sutherland, D., Overton, J.A., Matentzoglu, N., Balhoff, J., Harris, N.,

584 Brush, M., Touré, V., Sinclair, M., Poelen, J., Bretaudeau, A., Cain, S., Haendel, M.,

585 Vasilevsky, N., Hammock, J., Laporte, M.-A., Jensen, M. \& Larralde, M. (2021) oborel/obo-

586 relations: 2021-03-08 Release. Zenodo. https://doi.org/10.5281/zenodo.4589530

588 Mungall, C.J., Torniai, C., Gkoutos, G.V., Lewis, S.E. \& Haendel, M.A. (2012) Uberon, an

$589 \quad$ integrative multi-species anatomy ontology. Genome Biology 13, R5.

$590 \quad$ https://doi.org/10.1186/gb-2012-13-1-r5 
592 Musen, M.A. (2015) The Protégé project: a look back and a look forward. AI Matters 1, 4-12.

$593 \quad$ https://doi.org/10.1145/2757001.2757003

595 Natale, D.A, Arighi, C.N., Blake, J.A., Bona, J., Chen, C., Chen, S.-C., Christie, K.R., Cowart, 596 J., D’Eustachio, P., Diehl, A.D., Drabkin, H.J., Duncan, W.D., Huang, H., Ren, J., Ross, K., 597 Ruttenberg, A., Shamovsky, V., Smith, B., Wang, Q., Zhang, J., El-Sayed, A., Wu, C.H. 598 (2017) Protein Ontology (PRO): enhancing and scaling up the representation of protein 599 entities. Nucleic Acids Research 45: D339-D346. https://doi.org/10.1093/nar/gkw1075

601 Osumi-Sutherland, D., Marygold, S.J., Millburn, G.H., McQuilton, P.A., Ponting, L., Stefancsik, 602 R., Falls, K., Brown, N.H. \& Gkoutos, G.V. (2013) The Drosophila phenotype ontology. 603 Journal of Biomedical Semantics 4, 30. https://doi.org/10.1186/2041-1480-4-30

Page R.D.M. (2013) BioNames: linking taxonomy, texts, and trees. PeerJ, 1: e190.

Page, R.E. \& Amdam, G.V. (2007) The making of a social insect: developmental architectures of social design. BioEssays 29, 334-343. https://doi.org/10.1002/bies.20549

611 Penev, L., Lyal, C., Weitzman, A., Morse, D., King, D., Sautter, G., Georgiev, T., Morris, R., 612 Catapano, T. \& Agosti, D. (2011) XML schemas and mark-up practices of taxonomic 613 literature. ZooKeys 150: 89-116. https://doi.org/10.3897/zookeys.150.2213 
615 Pustejovsky. J. \& Stubbs, A. (2012) Natural Language Annotation for Machine Learning: A

616 Guide to Corpus-Building for Applications. O’Reilly Media, Inc., 342 pp.

617 https://www.google.com/books/edition/Natural_Language_Annotation_for_Machine/A57TS

$618 \quad \underline{7 \mathrm{fs} 8 \mathrm{MUC} ? \mathrm{hl}=\mathrm{en} \& \mathrm{gbpv}=0}$

619

620 Richards, A.G. \& Richards, P.A. (1979) The cuticular protuberances of insects. International

621 Journal of Insect Morphology and Embryology 8, 143-157. https://doi.org/10.1016/0020-

$622 \quad \underline{7322(79) 90013-8}$

623

624 Sautter, G., Böhm, K., Agosti, D. (2007) Semi-automated xml markup of biosystematic legacy

625 literature with the GoldenGATE editor. Pacific Symposium on Biocomputing, 12: 391-402.

$626 \underline{\text { https://doi.org/10.1142/9789812772435_0037 }}$

627

628 Smith, B., Ceusters, W., Klagges, B., Köhler, J., Kumar, A., Lomax, J., Mungall, C., Neuhaus,

629 F., Rector, A.L. \& Rosse, C. (2005) Relations in biomedical ontologies. Genome Biology 6,

630 R46. https://doi.org/10.1186/gb-2005-6-5-r46

631

632 Smith B., Ashburner M., Rosse C., Bard J., Bug W., Ceusters W., Goldberg L.J., Eilbeck K.,

633 Ireland A., Mungall C.J., Leontis N., Rocca-Serra P., Ruttenberg A., Sansone S.-A.,

634 Scheuermann R.H., Shah N., Whetzel P.L., Lewis S. (2007) The OBO Foundry: coordinated

635 evolution of ontologies to support biomedical data integration. Nature Biotechnology, 25:

636 1251-1255. https://doi.org/10.1038/nbt1346 
638 Snodgrass, R.E. (1935) Principles of Insect Morphology. Cornell University Press, 768 pp.

$639 \quad$ https://www.jstor.org/stable/10.7591/j.ctv1nhm1j

640

641 Snodgrass, R.E. (1951) Anatomy and Morphology. Journal of the New York Entomological

642 Society 59, 71-73. https://www.jstor.org/stable/25005398

643

644 Snodgrass, R.E. (1963) A contribution toward an encyclopedia of insect anatomy. Smithsonian

645 Miscellaneous Collections 146, 1-48. https://www.biodiversitylibrary.org/page/30259679

646

647 Sommer, R.J. (2020) Phenotypic plasticity: from theory and genetics to current and future

648 challenges. Genetics 215, 1-13. https://doi.org/10.1534/genetics.120.303163

650 Spear, A.D., Ceusters, W. \& Smith, B. (2016) Functions in Basic Formal Ontology. Applied

Ontology 11, 103-128. https://doi.org/10.3233/AO-160164

652

653 Stork N.E. (2018) How many species of insects and other terrestrial arthropods are there on

654 Earth? Annual Review of Entomology, 63: 31-45. https://doi.org/10.1146/annurev-ento-

655 $\underline{020117-043348}$

656

657 Stucky, B., Balhoff, J., Barve, N., Barve, V., Brenskelle, L., Brush, M., Dahlem, G., Gilbert, J., 658 Kawahara, A., Keller, O., Lucky, A., Mayhew, P., Plotkin, D., Seltmann, K., Talamas, E., 659 Vaidya, G., Walls, R., Yoder, M., Zhang, G. \& Guralnick, R. (2019) Developing a 
vocabulary and ontology for modeling insect natural history data: example data, use cases,

661 and competency questions. Biodiversity Data Journal 7, e33303.

662

https://doi.org/10.3897/BDJ.7.e33303

663

664 Tajiri, R. (2017) Cuticle itself as a central and dynamic player in shaping cuticle. Current

665 Opinion in Insect Science 19, 30-35. https://doi.org/10.1016/j.cois.2016.10.009

666

667 Tarasov, S. (2019) Integration of Anatomy Ontologies and Evo-Devo Using Structured Markov

668 Models Suggests a New Framework for Modeling Discrete Phenotypic Traits. Systematic

669 Biology 68, 698-716. https://doi.org/10.1093/sysbio/syz005

670

671 Topalis, P., Tzavlaki, C., Vestaki, K., Dialynas, E., Sonenshine, D.E., Butler, R., Bruggner, R.V.,

672 Stinson, E.O., Collins, F.H. \& Louis, C. (2008) Anatomical ontologies of mosquitoes and

673 ticks, and their web browsers in VectorBase. Insect Molecular Biology 17, 87-89.

$674 \quad$ https://doi.org/10.1111/j.1365-2583.2008.00781.x

675

676 Vogt, L. (2017) Assessing similarity: on homology, characters and the need for a semantic

677 approach to non-evolutionary comparative homology. Cladistics 33, 513-539.

$678 \quad$ https://doi.org/10.1111/cla.12179

679

680 Vogt, L. (2019) Levels and building blocks - toward a domain granularity framework for the life 681 sciences. Journal of Biomedical Semantics 10, 4. https://doi.org/10.1186/s13326-019-0196-2 
683 Vogt, L., Bartolomaeus, T. \& Giribet, G. (2010) The linguistic problem of morphology: structure

684 versus homology and the standardization of morphological data. Cladistics 26, 301-325.

$685 \quad \underline{\text { https://doi.org/10.1111/j.1096-0031.2009.00286.x }}$

686

687 Wagner, G.P. (2014) Homology, Genes, and Evolutionary Innovation. Princeton, New Jersey, $688 \quad$ United States, $496 \mathrm{pp}$.

689 https://press.princeton.edu/books/hardcover/9780691156460/homology-genes-and-

$690 \quad$ evolutionary-innovation

691

692 Wang, M., Li, Z. \& Zhou, Z. (2017) Insight into the growth pattern and bone fusion of basal

693 birds from an Early Cretaceous enantiornithine bird. Proceedings of the National Academy of Sciences 114, 11470-11475. https://doi.org/10.1073/pnas.1707237114

695

Washington, N.L., Haendel, M.A., Mungall, C.J., Ashburner, M., Westerfield, M. \& Lewis, S.E. (2009) Linking human diseases to animal models using ontology-based phenotype annotation. PLOS Biology 7, e1000247. https://doi.org/10.1371/journal.pbio.1000247

Wirkner, C.S., Göpel, T., Runge, J., Keiler, J., Klussmann-Fricke, B.-J., Huckstorf, K., Scholz, 701 S., Mikó, I., J. Yoder, M. \& Richter, S. (2017) The first organ-based ontology for arthropods 702 (Ontology of Arthropod Circulatory Systems - OArCS) and its integration into a novel 703 formalization scheme for morphological descriptions. Systematic Biology 66, 754-768.

$704 \quad$ https://doi.org/10.1093/sysbio/syw108 
706 Yoder, M.J., Mikó, I., Seltmann, K.C., Bertone, M.A. \& Deans, A.R. (2010) A Gross Anatomy

707 Ontology for Hymenoptera. PLOS ONE 5, e15991.

$708 \quad$ https://doi.org/10.1371/journal.pone.0015991

709

710 Yoshizawa, K. (2005) Morphology of Psocomorpha (Psocodea: 'Psocoptera'). Insecta

711 matsumurana. New series: journal of the Faculty of Agriculture Hokkaido University, series

712 entomology 62, 1-44. http://hdl.handle.net/2115/10524

713

714 Young, B.A. (1993) On the necessity of an archetypal concept in morphology: With special

715 reference to the concepts of "structure" and "homology." Biology and Philosophy 8, 225-

716 248. https://doi.org/10.1007/BF00850483

717

718 Zartman, J.J. \& Shvartsman, S.Y. (2010) Unit Operations of Tissue Development: Epithelial

719 Folding. Annual Review of Chemical and Biomolecular Engineering 1, 231-246.

720 https://doi.org/10.1146/annurev-chembioeng-073009-100919

721

722 Zhao, X., Gou, X., Qin, Z., Li, D., Wang, Y., Ma, E., Li, S. \& Zhang, J. (2017) Identification and

723 expression of cuticular protein genes based on Locusta migratoria transcriptome. Scientific

724 Reports 7, 45462. https://doi.org/10.1038/srep45462

725

726

$727 \quad$ Figure captions 
728 Figure 1. Autofluorescence-based CLSM micrograph showing the general structure of a sagittal

729 section of the insect integument in an adult treehopper, genus Ceresa sp. (Membracidae).

730 Excitation wavelength: 488, emission wavelengths: 500-580 pseudocolor green for conjunctivae,

731 muscles, and other soft structures and 580-700 pseudocolor red for sclerotized components.

732 Abbreviations: sc: sclerite; co: conjunctiva; sm: skeletal muscle; ap: appendage; in: invagination;

733 ev: evagination.

734

735 Figure 2. Schematic representation of some of the structural components of MoDIAS: a

736 conceptual Model for Describing Insect Anatomical Structures. The two principal structural

737 properties that characterize the insect cuticle are: 1) degree of flexibility (y axis), ranging from

738 sclerite (stiff, at the top) to conjunctiva (flexible, at the bottom), and 2) degree of curvature ( $\mathrm{x}$

739 axis; protrusion -left- to flat -center- to depression -right-); the degree of curvature of the external

740 and internal surfaces of cuticular protrusions and depressions can be different: when both run in

741 parallel, they form hollow protrusions (top left subplane, e.g., carina, tubercle) or hollow

742 depressions (top right subplane, yellow band e.g., pit, sulcus); the external surface can be

743 depressed with the internal surface flat (top right subplane, purple band, e.g., fovea, groove); the

744 external surface can be flat with the internal surface depressed (top right subplane, blue band,

745 e.g., ridge, apodeme). Additional properties: quantity (single vs. multiple elements; pink and

746 orange bands, respectively); shape can be observed throughout each subplane (e.g., sclerotized

747 protrusions can range from elongated - carina- to rounded -tubercle-); same for depressions

748 (elongate -groove- vs. rounded -fovea-).

749 
750 Figure 3. Schematic representation of taxon-specific definitions for paired cuticular structures of

751 the abdominal tergites (not including structures of the genitalia) across different insect orders.

752 Textual definitions for each structure are provided in Table 3.

753

754 Figure 4. Graphic representation of high-level classes in AISM and some of their children, 755 including hierarchy and elements from other ontologies. 